TRANSACTIONS OF THE

AMERICAN MATHEMATICAL SOCIETY

Volume 355, Number 2, Pages 813-836

S 0002-9947(02)03148-3

Article electronically published on October 9, 2002

\title{
NOTES ON INTERPOLATION IN THE GENERALIZED SCHUR CLASS. II. NUDEL'MAN'S PROBLEM
}

\author{
D. ALPAY, T. CONSTANTINESCU, A. DIJKSMA, AND J. ROVNYAK
}

\begin{abstract}
An indefinite generalization of Nudel'man's problem is used in a systematic approach to interpolation theorems for generalized Schur and Nevanlinna functions with interior and boundary data. Besides known results on existence criteria for Pick-Nevanlinna and Carathéodory-Fejér interpolation, the method yields new results on generalized interpolation in the sense of Sarason and boundary interpolation, including properties of the finite Hilbert transform relative to weights. The main theorem appeals to the Ball and Helton almost-commutant lifting theorem to provide criteria for the existence of a solution to Nudel'man's problem.
\end{abstract}

\section{INTRODUCTION}

In this paper we use a theorem of Ball and Helton [11] to describe a unified approach to a series of classical interpolation problems for the generalized Schur and Nevanlinna classes. These classes contain meromorphic functions having a finite number of poles on a disk or half-plane. The main results of the paper update Chapter 2 of [29], where similar results are derived for holomorphic functions.

In [29], classical interpolation problems are viewed as special cases of Nudel'man's problem: given vectors $b$ and $c$ in a complex vector space $\mathcal{V}$ and an operator $A$ on $\mathcal{V}$ it is required to find a holomorphic function $f(z)$ which is bounded by one on the unit disk such that

$$
b=f(A) c
$$

with a suitable interpretation of the equation. Particular choices of $A, b$, and $c$ give various classical problems, such as the Pick-Nevanlinna and Carathéodory-Fejér problems and boundary problems of Loewner type. Conditions for the existence of a solution of Nudel'man's problem are derived in [29, Chapter 2] as an application of the theory of contraction operators on a Hilbert space. This yields existence criteria for classical interpolation problems for Schur and Nevanlinna functions, that is, holomorphic functions which are bounded by one on the unit disk or which have nonnegative imaginary part on the upper half-plane. However, the scope of the method does not include interpolation at a finite number of boundary points (for example, see Sarason [34]).

Received by the editors September 18, 2001 and, in revised form, April 16, 2002.

2000 Mathematics Subject Classification. Primary 47A57, 30E05, 47B32; Secondary 47B50, $42 \mathrm{~A} 50$.

J. Rovnyak was supported by the National Science Foundation DMS-0100437 and by the Netherlands Organization for Scientific Research NWO B 61-482.

(C)2002 American Mathematical Society 
Our main tool is an indefinite generalization of Nudel'man's problem, which provides a similar framework for interpolation problems involving the classes $\mathbf{S}_{\kappa}$ and $\mathbf{N}_{\kappa}$ of generalized Schur and Nevanlinna functions on the unit disk and upper half-plane. These well-known classes consist of meromorphic functions having a finite number of poles. As in the definite case, we derive existence criteria for the solvability of the generalized Nudel'man problem in an abstract setting, and the applications to classical problems follow as specializations of this result.

The main results on interpolation in the generalized Schur and Nevanlinna classes are known. Classical sources and connections with the Takagi and Nehari problems may be found in the seminal work of Adamjan, Arov, and Krein [1]. The approach by means of invariant subspaces and commutant lifting is due to Ball and Helton [11. The list below identifies some more recent works that treat these topics and related areas. The list is not a complete bibliography, and the authors regret omissions.

Interpolation of interior data: [1], [4], [10], 11], [12, [16], 17], [19, 20], 22], 26], 31]

Loewner theory and boundary data: [5, 7], 11]

Parametrization of solutions: [1, [10], [11, [19]

Interpolation in the Stieltjes class, including parametrizations: [2, 8

Moment problems: [13, [14, 24]

Monotone operator functions: 3

Our generalization of Nudel'man's problem and Main Theorem are formulated in Section 2 Sections 3 and 4 contain the applications of the Main Theorem to classical interpolation problems in the disk and half-plane cases, respectively; many of these results parallel the definite case [29]. The proof of the Main Theorem is given in Section 5. This paper can be read independently of [4].

After this paper was completed, the authors received a copy of Popescu [28], which applies similar methods in a several variable setting.

\section{MAIN THeOREM}

By a kernel or Hermitian form we mean a complex-valued function $K(\zeta, z)=$ $\overline{K(z, \zeta)}$ on a product set $\Omega \times \Omega$. Such a kernel has $\kappa$ negative squares, in symbols sq_ $K=\kappa$, if every selfadjoint matrix $\left(K\left(\zeta_{j}, \zeta_{i}\right)\right)_{i, j=1}^{n}, \zeta_{1}, \ldots, \zeta_{n} \in \Omega$, $n=1,2,3, \ldots$, has at most $\kappa$ negative eigenvalues, and one such matrix has exactly $\kappa$ negative eigenvalues (counting multiplicity). When this condition is satisfied with $\kappa=0$, the kernel is said to be nonnegative. A familiar example of a Hermitian form is the inner product $\langle T f, g\rangle_{\mathfrak{H}}$, where $T$ is a bounded selfadjoint operator on a Hilbert space $\mathfrak{H}$ and $f$ and $g$ are arbitrary vectors in $\mathfrak{H}$. We say that $T$ is nonnegative or has $\kappa$ negative squares according as the associated Hermitian form has the same property.

The Pick class or Nevanlinna class is the set $\mathcal{P}$ of holomorphic functions which have nonnegative imaginary part on the open upper half-plane $\mathbf{C}_{+}$. The Schur class is the set $\mathcal{S}$ of holomorphic functions which are bounded by one on the open unit disk D. Let $\mathbf{C}$ be the complex plane. Following KreĬn and Langer [24], for any nonnegative integer $\kappa$ we define the generalized Nevanlinna class as the set $\mathbf{N}_{\kappa}$ of functions $f(z)$ which are holomorphic on some subregion $\Omega$ of $\mathbf{C}_{+}$ 
such that the kernel

$$
\frac{f(z)-\overline{f(\zeta)}}{z-\bar{\zeta}}
$$

has $\kappa$ negative squares on $\Omega \times \Omega$. The generalized Schur class is the set $\mathbf{S}_{\kappa}$ of functions $S(z)$ which are holomorphic on some subregion $\Omega$ of $\mathbf{D}$ such that the kernel

$$
\frac{1-S(z) \overline{S(\zeta)}}{1-z \bar{\zeta}}
$$

has $\kappa$ negative squares on $\Omega \times \Omega$. Functions in the generalized Nevanlinna and Schur classes have analytic continuations to $\mathbf{C}_{+}$and $\mathbf{D}$, respectively, excluding at most $\kappa$ poles, and the corresponding kernels for the extensions also have $\kappa$ negative squares. We understand that such functions are identified with their meromorphic continuations. With this convention, $\mathbf{N}_{0}=\mathcal{P}$ and $\mathbf{S}_{0}=\mathcal{S}$. By a theorem of Kreln and Langer [23, Theorem 3.2 on p. 382], $\mathbf{S}_{\kappa}$ coincides with the set of functions

$$
S(z)=f(z) / B(z), \quad B(z)=c \prod_{j=1}^{\kappa} \frac{z-a_{j}}{1-z \bar{a}_{j}}
$$

where $f(z)$ is in $\mathbf{S}_{0}, a_{1}, \ldots, a_{\kappa}$ are (not necessarily distinct) points in $\mathbf{D},|c|=1$, and $f\left(a_{j}\right) \neq 0$ for all $j=1, \ldots, \kappa$. A function $B(z)$ on the unit disk of the preceding form is a Blaschke product of degree $\kappa$. We call (2.1) a KreйnLanger factorization of a function $S \in \mathbf{S}_{\kappa}$. The factors $f(z)$ and $B(z)$ in a Kreı̆n-Langer factorization are determined up to constants of modulus one.

The original inspiration for what is called Nudel'man's problem in [29, p. 22] is Nudel'man 25. We now consider the following variant.

Nudel'man's Problem for $\mathbf{S}_{\kappa}$. Given vectors $b, c$ in a complex vector space $\mathcal{V}$ and a linear operator $A$ on $\mathcal{V}$ into itself, find a pair $(f, B)$, where $f \in \mathbf{S}_{0}$ and $B$ is a Blaschke product of degree $\kappa$, such that

$$
f(A) c=B(A) b .
$$

We call $(A, b, c)$ the data of the problem.

Here and in the rest of this section we assume that we are given a complex vector space $\mathcal{V}$ with algebraic dual $\mathcal{V}^{\prime}$ (no topologies on these spaces are required). If $x^{\prime} \in \mathcal{V}^{\prime}$, we write $\left(x, x^{\prime}\right)$ for $x^{\prime}(x)$. If $A: \mathcal{V} \rightarrow \mathcal{V}$ is a linear operator, its dual $A^{\prime}$ is the operator $A^{\prime}: \mathcal{V}^{\prime} \rightarrow \mathcal{V}^{\prime}$ such that $\left(x, A^{\prime} x^{\prime}\right)=\left(A x, x^{\prime}\right)$ for all $x \in \mathcal{V}$ and $x^{\prime} \in \mathcal{V}^{\prime}$.

Definition 2.1. A set $\mathcal{D} \subseteq \mathcal{V}^{\prime}$ is admissible for given data $(A, b, c)$ if

(i) $\mathcal{D}$ is a linear subspace of $\mathcal{V}^{\prime}$ which is invariant under $A^{\prime}$,

(ii) the sums $\sum_{j=0}^{\infty}\left|\left(A^{j} b, x^{\prime}\right)\right|^{2}$ and $\sum_{j=0}^{\infty}\left|\left(A^{j} c, x^{\prime}\right)\right|^{2}$ are finite for all $x^{\prime} \in \mathcal{D}$, and

(iii) if some $x^{\prime}$ in $\mathcal{D}$ annihilates $c, A c, A^{2} c, \ldots$, then $x^{\prime}$ annihilates $b, A b, A^{2} b, \ldots$

Throughout we write $H^{2}$ for the Hardy space on the unit disk.

Definition 2.2. If $\mathcal{D}$ is admissible for $(A, b, c)$, then for any $f(z)=\sum_{j=0}^{\infty} f_{j} z^{j}$ and $B(z)=\sum_{j=0}^{\infty} B_{j} z^{j}$ in $H^{2}$, we interpret (2.2) to mean that

$$
\sum_{j=0}^{\infty} f_{j}\left(A^{j} c, x^{\prime}\right)=\sum_{j=0}^{\infty} B_{j}\left(A^{j} b, x^{\prime}\right), \quad x^{\prime} \in \mathcal{D} .
$$


Thus the meaning of (2.2) depends on the choice of an admissible set $\mathcal{D}$ of linear functionals. It will be seen that in applications natural choices are usually clear.

Main Theorem. Let $(A, b, c)$ be given data and $\mathcal{D}$ an admissible set. Define a Hermitian form $\mathcal{F}$ on $\mathcal{D} \times \mathcal{D}$ by

$$
\mathcal{F}\left(x^{\prime}, y^{\prime}\right)=\sum_{j=0}^{\infty}\left[\left(A^{j} c, x^{\prime}\right) \overline{\left(A^{j} c, y^{\prime}\right)}-\left(A^{j} b, x^{\prime}\right) \overline{\left(A^{j} b, y^{\prime}\right)}\right], \quad x^{\prime}, y^{\prime} \in \mathcal{D} .
$$

Let $\kappa$ be a nonnegative integer.

(1) If $\mathcal{F}$ has $\kappa$ negative squares, there is a pair $(f, B)$, where $f \in \mathbf{S}_{0}$ and $B$ is a Blaschke product of degree $\kappa$, such that $f(A) c=B(A) b$.

(2) If there is a pair $(f, B)$ as in $(1)$, then $\mathcal{F}$ has $\kappa^{\prime}$ negative squares for some $\kappa^{\prime} \leq \kappa$.

We show that in the case $\kappa=0$, this result implies Theorem 2.3 in 29. The reason there is something to show is that we now require condition (iii) in Definition 2.1] as a hypothesis, and this is not a hypothesis in [29, Theorem 2.3, p. 23]. It is therefore sufficient to show that when $\kappa=0$, condition (iii) in Definition [2.1] is redundant when the other hypotheses in parts (1) and (2) of the Main Theorem are satisfied.

(1) Assume the hypotheses of part (1) of the Main Theorem with $\kappa=0$, but not necessarily condition (iii) in Definition 2.1. Then $\mathcal{F}$ is nonnegative, and we have the inequality

$$
\sum_{j=0}^{\infty}\left|\left(A^{j} b, x^{\prime}\right)\right|^{2} \leq \sum_{j=0}^{\infty}\left|\left(A^{j} c, x^{\prime}\right)\right|^{2}, \quad x^{\prime} \in \mathcal{D},
$$

which implies (iii).

(2) Assume the hypotheses of part (2) of the Main Theorem with $\kappa=0$, but not necessarily condition (iii) in Definition 2.1 Then $B$ is a constant of absolute value one, and we may take $B \equiv 1$. Thus

$$
\sum_{j=0}^{\infty} f_{j}\left(A^{j} c, x^{\prime}\right)=\left(b, x^{\prime}\right), \quad x^{\prime} \in \mathcal{D} .
$$

If some $x_{0}^{\prime}$ in $\mathcal{D}$ annihilates $c, A c, A^{2} c, \ldots$, then by Definition 2.1 (i), for all $k=$ $0,1,2, \ldots,\left(A^{\prime}\right)^{k} x_{0}^{\prime}$ also belongs to $\mathcal{D}$ and annihilates $c, A c, A^{2} c, \ldots$, and hence $\left(b,\left(A^{\prime}\right)^{k} x_{0}^{\prime}\right)=0$ by (2.5). Thus $x_{0}^{\prime}$ annihilates $b, A b, A^{2} b, \ldots$, and we again obtain Definition 2.1(iii).

The role of condition (iii) in Definition 2.1 can be seen in the applications in Section 3. For example, in Theorem 3.1 the condition follows from the assumption that the points $z_{1}, \ldots, z_{n}$ are distinct; this assumption is redundant in classical Pick-Nevanlinna interpolation in the case $\kappa=0$.

The proof of the Main Theorem is given in Section 5 .

\section{Classical interpolation Problems on the Unit Disk}

We begin with the prototype for classical interpolation theory, Pick-Nevanlinna interpolation at a finite number of points. 
Theorem 3.1. Let $z_{1}, \ldots, z_{n}$ be distinct points in $\mathbf{D}, w_{1}, \ldots, w_{n}$ any complex numbers, and let $\kappa$ be a nonnegative integer. Set

$$
P=\left[\frac{1-w_{j} \bar{w}_{k}}{1-z_{j} \bar{z}_{k}}\right]_{j, k=1}^{n} .
$$

(1) If $P$ has $\kappa$ negative eigenvalues, then there is a pair $(f, B)$ with $f \in \mathbf{S}_{0}$ and $B$ a Blaschke product of degree $\kappa$ such that $f\left(z_{j}\right)=B\left(z_{j}\right) w_{j}$ for all $j=1, \ldots, n$.

(2) If there is a pair $(f, B)$ as in (1), then $P$ has $\kappa^{\prime} \leq \kappa$ negative eigenvalues.

Proof. We apply the Main Theorem with $\mathcal{V}=\mathbf{C}^{n}$. Identify $\mathcal{V}^{\prime}$ with $\mathbf{C}^{n}$ with the pairing $(x, y)=x_{1} y_{1}+\cdots+x_{n} y_{n}$ (for any $a$ in $\mathbf{C}^{n}$, we write $a_{j}$ for its entries). For the data $(A, b, c)$, choose

$$
A=\operatorname{diag}\left\{z_{1}, \ldots, z_{n}\right\}, \quad b=\left[\begin{array}{c}
w_{1} \\
\vdots \\
w_{n}
\end{array}\right], \quad c=\left[\begin{array}{c}
1 \\
\vdots \\
1
\end{array}\right],
$$

and take $\mathcal{D}=\mathcal{V}^{\prime}$. The verification that $\mathcal{D}$ is admissible is routine. To check property (iii) in Definition 2.1 suppose $x \in \mathcal{D}$ annihilates $c, A c, A^{2} c, \ldots$ Then

$$
\sum_{p=1}^{n} z_{p}^{j} x_{j}=0, \quad j \geq 0 .
$$

Since $z_{1}, \ldots, z_{n}$ are distinct, $x=0$ and trivially $x$ annihilates $b, A b, A^{2} b, \ldots$. A short calculation shows that the Hermitian form (2.4) is given by

$$
\mathcal{F}(x, y)=\sum_{j, k=1}^{n} \frac{1-w_{j} \bar{w}_{k}}{1-z_{j} \bar{z}_{k}} x_{j} \bar{y}_{k}, \quad x, y \in \mathcal{D} .
$$

Thus sq_ $\mathcal{F}$ is equal to the number of negative eigenvalues of $P$. To see the meaning of the identity $B(A) b=f(A) c$, in (2.3) choose the linear functional induced by the standard unit vector with 1 in the $k$-th entry and all other entries zero. In this case the condition reduces to $B\left(z_{k}\right) w_{k}=f\left(z_{k}\right)$. Thus the result is a particular case of the Main Theorem.

With a slight variation of method, the preceding result can be extended to an arbitrary set of points.

Theorem 3.2. Let $S_{0}: \Omega \rightarrow \mathbf{C}$ be a function defined on a subset $\Omega$ of $\mathbf{D}$, and let $\kappa$ be a nonnegative integer. Set

$$
K_{0}(\zeta, z)=\frac{1-S_{0}(z) \overline{S_{0}(\zeta)}}{1-z \bar{\zeta}}, \quad \zeta, z \in \Omega .
$$

(1) If $\mathrm{sq}_{-} K_{0}=\kappa$, then there is a pair $(f, B)$ with $f \in \mathbf{S}_{0}$ and $B$ a Blaschke product of degree $\kappa$ such that $f(z)=B(z) S_{0}(z)$ for all $z \in \Omega$.

(2) If there is a pair $(f, B)$ as in (1), then $\mathrm{sq}_{-} K_{0}=\kappa^{\prime} \leq \kappa$.

Proof. Let $\mathcal{V}$ be the vector space of all functions $x: \Omega \rightarrow \mathbf{C}$. For the data $(A, b, c)$, let $A$ be multiplication by the independent variable on $\mathcal{V}, b=S_{0}$, and $c \equiv 1$. Every function $y$ on $\Omega$ with finite support induces a linear functional on $\mathcal{V}$ by the formula $(x, y)=\sum_{z \in \Omega} x(z) y(z)$, and the set $\mathcal{D}$ of all such functionals is admissible. A 
straightforward application of the Main Theorem as in the proof of Theorem 3.1 yields the result.

Theorems 3.1 and 3.2 imply interpolation results for the class $\mathbf{S}_{\kappa}$. Thus in Theorem 3.2 we can divide the relation $f(z)=B(z) S_{0}(z), z \in \Omega$, by the Blaschke factor. Since $B$ can have zeros at the interpolating points, we do not necessarily obtain interpolation at all points.

Corollary 3.3. Let $S_{0}: \Omega \rightarrow \mathbf{C}$ be a function defined on a subset $\Omega$ of $\mathbf{D}$, and let $\kappa$ be a nonnegative integer. If the kernel $K_{0}(\zeta, z)$ defined in Theorem 3.2 has $\kappa$ negative squares, then there is a $\kappa^{\prime} \leq \kappa$ and a function $S$ in $\mathbf{S}_{\kappa^{\prime}}$ such that $S(z)=S_{0}(z)$ for all $z \in \Omega$ with the possible exception of up to $\kappa$ points.

The next result concerns the interpolation of a finite number of derivatives, that is, it is of the Carathéodory-Fejér type. We use standard notation for matrices and their induced operators on Euclidean spaces. Write 1 for an identity matrix or operator and * for conjugate transpose or adjoint.

Theorem 3.4. Let $w(z)=w_{0}+w_{1} z+\cdots+w_{n} z^{n}$ be a polynomial with complex coefficients, and set

$$
T=\left[\begin{array}{cccc}
w_{0} & w_{1} & \cdots & w_{n} \\
0 & w_{0} & \cdots & w_{n-1} \\
& & \cdots & \\
0 & 0 & \cdots & w_{0}
\end{array}\right] .
$$

Let $\kappa$ be a nonnegative integer such that $\kappa \leq n+1$.

(1) If $1-T^{*} T$ has $\kappa$ negative eigenvalues, then there is a pair $(f, B)$ with $f \in \mathbf{S}_{0}$ and $B$ a Blaschke product of degree $\kappa$ such that $B(z) w(z)=f(z)+\mathcal{O}\left(z^{n+1}\right)$.

(2) If there is a pair $(f, B)$ as in (1), then $1-T^{*} T$ has $\kappa^{\prime} \leq \kappa$ negative eigenvalues.

Corollary 3.5. Let $w(z)=w_{0}+w_{1} z+\cdots+w_{n} z^{n}$, let $T$ be as in Theorem 3.4, and let $\kappa$ be a nonnegative integer such that $\kappa \leq n+1$. If $1-T^{*} T$ has $\kappa$ negative eigenvalues, then there is a $\kappa^{\prime} \leq \kappa$ and a function $S(z)$ in $\mathbf{S}_{\kappa^{\prime}}$ which is holomorphic at the origin and such that $w(\bar{z})=S(z)+\mathcal{O}\left(z^{n+1-\kappa}\right)$.

Proof of Theorem 3.4. Let $\mathcal{V}$ and $\mathcal{V}^{\prime}$ be as in the proof of Theorem 3.1 but with $\mathbf{C}^{n}$ replaced by $\mathbf{C}^{n+1}$. For the data $(A, b, c)$, choose

$$
A=\left[\begin{array}{ccccc}
0 & 0 & \cdots & 0 & 0 \\
1 & 0 & \cdots & 0 & 0 \\
0 & 1 & \cdots & 0 & 0 \\
& & \cdots & & \\
0 & 0 & \cdots & 1 & 0
\end{array}\right], \quad b=\left[\begin{array}{c}
w_{0} \\
w_{1} \\
\vdots \\
w_{n}
\end{array}\right], \quad c=\left[\begin{array}{c}
1 \\
0 \\
\vdots \\
0
\end{array}\right] .
$$

The set $\mathcal{D}=\mathcal{V}^{\prime}$ is admissible. If $\mathfrak{H}$ is $\mathbf{C}^{n+1}$ in the Euclidean inner product, then $\mathcal{F}(x, y)=\left\langle\left(1-T^{*} T\right) x, y\right\rangle_{\mathfrak{H}}, x, y \in \mathfrak{H}$. In fact, for any $x \in \mathfrak{H}$,

$$
\begin{aligned}
\mathcal{F}(x, x)= & \left(\left|x_{0}\right|^{2}+\cdots+\left|x_{n}\right|^{2}\right) \\
& \quad-\left(\left|w_{0} x_{0}+\cdots+w_{n} x_{n}\right|^{2}+\left|w_{0} x_{1} \cdots+w_{n-1} x_{n}\right|^{2}+\cdots+\left|w_{0} x_{n}\right|^{2}\right) \\
= & \|x\|_{\mathfrak{H}}^{2}-\|T x\|_{\mathfrak{H}}^{2} \\
= & \left\langle\left(1-T^{*} T\right) x, x\right\rangle_{\mathfrak{H}} .
\end{aligned}
$$


Thus sq_ $\mathcal{F}$ is equal to the number of negative eigenvalues of $1-T^{*} T$. The equation $f(A) c=B(A) b$ with $f(z)=\sum_{j=0}^{\infty} f_{j} z^{j}$ and $B(z)=\sum_{j=0}^{\infty} B_{j} z^{j}$ is equivalent to the identities

$$
f_{0}=w_{0} B_{0}, f_{1}=w_{1} B_{0}+w_{0} B_{1}, \ldots, f_{n}=w_{n} B_{0}+w_{n-1} B_{1}+\cdots+w_{0} B_{n},
$$

or $B(z) w(z)=f(z)+\mathcal{O}\left(z^{n+1}\right)$. The result thus follows from the Main Theorem.

Proof of Corollary 3.5. Let $(f, B)$ be a pair as in part (1) of Theorem 3.4. If $B(z)$ has a zero of order $r$ at the origin, then $f(z)$ has a zero of order at least $r$ at the origin. Hence $S(z)=f(z) / B(z)$ belongs to $\mathbf{S}_{\kappa^{\prime}}$ for some $\kappa^{\prime} \leq \kappa$ and is holomorphic at the origin, and $w(z)=S(z)+\mathcal{O}\left(z^{n-r+1}\right)=S(z)+\mathcal{O}\left(z^{n+1-\kappa}\right)$.

A simultaneous generalization of the Pick-Nevanlinna and Carathéodory-Fejér problems can be treated in the same way by choosing $A$ in Jordan form. The calculations are straightforward but somewhat lengthy, and we shall not pursue this direction. For the definite case, see [21] and [29, §2.6].

The Main Theorem also yields a result on generalized interpolation in the sense of Sarason [32. Let $C$ be an inner function on $\mathbf{D}$, and let $\mathfrak{H}(C)=H^{2} \ominus C H^{2}$. The reproducing kernel for $\mathfrak{H}(C)$ is given by

$$
K_{C}(w, z)=\frac{1-C(z) \overline{C(w)}}{1-z \bar{w}}, \quad z, w \in \mathbf{D}
$$

Let $S$ be the shift operator $S: h(z) \rightarrow z h(z)$ on $H^{2}$, and let $T$ be the compression of $S$ to $\mathfrak{H}(C)$, that is,

$$
T=\left.P_{\mathfrak{H}(C)} S\right|_{\mathfrak{H}(C)},
$$

where $P_{\mathfrak{H}(C)}$ is the projection operator on $H^{2}$ with range $\mathfrak{H}(C)$. The space $\mathfrak{H}(C)$ is invariant under $S^{*}$ and $T^{*}=\left.S^{*}\right|_{\mathfrak{H}(C)}$. Since $T$ is completely nonunitary, for any $\varphi \in H^{\infty}$, an operator $\varphi(T)$ on $\mathfrak{H}(C)$ is defined by the $H^{\infty}$-functional calculus (see [35] and [36, p. 114]):

$$
\varphi(T)=s-\lim _{r \uparrow 1} \varphi(r T) .
$$

Equivalently, for this particular situation, $\varphi(T)=\left.P_{\mathfrak{H}(C)} M_{\varphi}\right|_{\mathfrak{H}(C)}$, where $M_{\varphi}$ is multiplication by $\varphi$ on $H^{2}$. For every $\varphi \in H^{\infty}, \varphi(T)$ commutes with $T$, and $\varphi(T)$ is a contraction if and only if $\varphi$ is a Schur function.

Theorem 3.6. Let $C$ be an inner function on the unit disk, and define $T$ on $\mathfrak{H}(C)$ as above. Let $R$ be a bounded linear operator on $\mathfrak{H}(C)$ such that $T R=R T$.

(1) If $1-R R^{*}$ has $\kappa$ negative squares, then there is a pair $(f, B)$, where $f \in \mathbf{S}_{0}$ and $B$ is a Blaschke product of degree $\kappa$, such that

$$
B(T) R=f(T) .
$$

(2) If there is a pair $(f, B)$ as in $(1)$, then $1-R R^{*}$ has $\kappa^{\prime}$ negative squares for some $\kappa^{\prime} \leq \kappa$.

If $R$ is a contraction, the condition in (1) is satisfied with $\kappa=0$, and in this case the result reduces to the original theorem of Sarason [32, Theorem 1].

Proof. In the Main Theorem, let $\mathcal{V}=\mathfrak{H}(C), A=T, c=K_{C}(0, \cdot)$, and $b=R c=$ $R K_{C}(0, \cdot)$. Let $\mathcal{D}$ be the set of continuous linear functionals on $\mathcal{V}=\mathfrak{H}(C)$; thus $\mathcal{D}=\left\{x_{k}^{\prime}: k \in \mathfrak{H}(C)\right\}$, where for any $k \in \mathfrak{H}(C)$,

$$
\left(h, x_{k}^{\prime}\right)=\langle h, k\rangle_{\mathfrak{H}(C)}, \quad h \in \mathfrak{H}(C) .
$$


Then $A^{\prime} x_{k}^{\prime}=x_{T^{*} k}^{\prime}$ for any $k \in \mathfrak{H}(C)$, and so condition (i) in Definition 2.1 holds. To verify (ii), notice that for any $k(z)=\sum_{0}^{\infty} a_{j} z^{j}$ in $\mathfrak{H}(C)$,

$$
\sum_{j=0}^{\infty}\left|\left(A^{j} c, x_{k}^{\prime}\right)\right|^{2}=\sum_{j=0}^{\infty}\left|\left\langle K_{C}(0, \cdot), T^{* j} k\right\rangle_{\mathfrak{H}(C)}\right|^{2}=\sum_{j=0}^{\infty}\left|a_{j}\right|^{2}=\|k\|_{H^{2}}^{2}<\infty .
$$

If we replace $c$ by $b$ and use the identity $R T=T R$, we obtain

$$
\sum_{j=0}^{\infty}\left|\left(A^{j} b, x_{k}^{\prime}\right)\right|^{2}=\sum_{j=0}^{\infty}\left|\left\langle K_{C}(0, \cdot), T^{* j} R^{*} k\right\rangle_{\mathfrak{H}(C)}\right|^{2}=\left\|R^{*} k\right\|_{H^{2}}^{2}<\infty,
$$

and thus condition (ii) in Definition 2.1 holds. Condition (iii) holds trivially because the only $k \in \mathfrak{H}(C)$ such that

$$
\left(A^{j} c, x_{k}^{\prime}\right)=\left\langle K_{C}(0, \cdot), T^{* j} k\right\rangle_{\mathfrak{H}(C)}=0
$$

for all $j \geq 0$ is $k=0$. We have shown that $\mathcal{D}$ is admissible. The form (2.4) is given by

$$
\begin{aligned}
\mathcal{F}\left(x_{h}^{\prime}, x_{k}^{\prime}\right)= & \sum_{j=0}^{\infty}\left[\left\langle T^{j} K_{C}(0, \cdot), h\right\rangle_{\mathfrak{H}(C)}\left\langle k, T^{j} K_{C}(0, \cdot)\right\rangle_{\mathfrak{H}(C)}\right. \\
& \left.\quad-\left\langle T^{j} R K_{C}(0, \cdot), h\right\rangle_{\mathfrak{H}(C)}\left\langle k, T^{j} R K_{C}(0, \cdot)\right\rangle_{\mathfrak{H}(C)}\right] \\
= & \langle k, h\rangle_{H^{2}}-\left\langle R^{*} k, R^{*} h\right\rangle_{H^{2}} \\
= & \langle k, h\rangle_{\mathfrak{H}(C)}-\left\langle R^{*} k, R^{*} h\right\rangle_{\mathfrak{H}(C)} \\
= & \left\langle\left(1-R R^{*}\right) k, h\right\rangle_{\mathfrak{H}(C)}
\end{aligned}
$$

for any $h$ and $k$ in $\mathfrak{H}(C)$.

(1) Assume that $1-R R^{*}$ has $\kappa$ negative squares. By (3.3), the Hermitian form (2.4) has $\kappa$ negative squares. Hence by part (1) of the Main Theorem, there is a function $f \in \mathbf{S}_{0}$ and a Blaschke product $B$ of degree $\kappa$ such that $B(A) b=f(A) c$ in the sense of Definition [2.2. that is, if $B(z)=\sum_{0}^{\infty} B_{j} z^{j}$ and $f(z)=\sum_{0}^{\infty} f_{j} z^{j}$, then for every $h \in \mathfrak{H}(C)$,

$$
\sum_{j=0}^{\infty} B_{j}\left\langle T^{j} R K_{C}(0, \cdot), h\right\rangle_{\mathfrak{H}(C)}=\sum_{j=0}^{\infty} f_{j}\left\langle T^{j} K_{C}(0, \cdot), h\right\rangle_{\mathfrak{H}(C)} .
$$

Using Abel summation of these series, we see that

$$
B(T) R K_{C}(0, \cdot)=f(T) K_{C}(0, \cdot) .
$$

Since $R$ commutes with $T$, it commutes with $B(T)$ and $f(T)$. Hence $B(T) R$ and $f(T)$ agree on the smallest invariant subspace of $T$ containing $K_{C}(0, \cdot)$. The latter subspace is all of $\mathfrak{H}(C)$, and thus we obtain $B(T) R=f(T)$.

(2) Assume that a pair $(f, B)$ exists as in (1). Reversing the preceding steps, we see that $B(A) b=f(A) c$; hence by part (2) of the Main Theorem, the form (2.4) has $\kappa$ negative squares. Therefore by (3.3), $1-R R^{*}$ has $\kappa^{\prime}$ negative squares for some $\kappa^{\prime} \leq \kappa$.

More generally, let $C$ be a function (not necessarily an inner function) which is holomorphic and bounded by one on $\mathbf{D}$, and let $\mathfrak{H}(C)$ be the Hilbert space with reproducing kernel (3.1) [15, 33]. Then $\mathfrak{H}(C)$ is contained contractively in $H^{2}$, that 
is, the inclusion mapping $E: \mathfrak{H}(C) \rightarrow H^{2}$ is a contraction operator (the inclusion is isometric if and only if $C$ is an inner function). There is a contraction operator $T$ on $\mathfrak{H}(C)$ such that $T^{*}: h(z) \rightarrow[h(z)-h(0)] / z$ for every $h(z)$ in $\mathfrak{H}(C)$. In view of the difference-quotient inequality

$$
\|[h(z)-h(0)] / z\|_{\mathfrak{H}(C)}^{2} \leq\|h(z)\|_{\mathfrak{H}(C)}^{2}-|h(0)|^{2},
$$

which holds for every element $h(z)$ of $\mathfrak{H}(C), T$ is completely nonunitary. Hence for any $\varphi \in H^{\infty}$ we may define $\varphi(T)$ by (3.2). The selfadjoint operators $G=E^{*} E$ on $\mathfrak{H}(C)$ and $D=E E^{*}$ on $H^{2}$ also play a role. They satisfy $0 \leq G \leq 1$ and $0 \leq D \leq 1$. It is not hard to see that

$$
D=1-M_{C} M_{C}^{*},
$$

where $M_{C}$ is multiplication by $C$ on $H^{2}$. Let

$$
\mathfrak{M}=H^{2} \ominus \operatorname{ker} D \text {. }
$$

Then $\mathfrak{H}(C)$ coincides with the range of $D^{1 / 2}$ in the "range norm", that is, the unique norm such that $D^{1 / 2}$ acts as a partial isometry from $H^{2}$ onto $\mathfrak{H}(C)$ with initial space $\mathfrak{M}$. One can use the relation $D E=E G$ to show that $G$ is unitarily equivalent to $\left.D\right|_{\mathfrak{M}}$ by means of the natural isomorphism $U=\left.D^{1 / 2}\right|_{\mathfrak{M}}$ from $\mathfrak{M}$ onto $\mathfrak{H}(C)$.

Theorem 3.7. Let $C$ be a function which is holomorphic and bounded by one on the unit disk, and let $T$ be the operator on $\mathfrak{H}(C)$ such that $T^{*}: h(z) \rightarrow[h(z)-h(0)] / z$ for every $h(z)$ in $\mathfrak{H}(C)$. Let $G=E^{*} E$ and $E$ be as above. Let $R$ be a bounded linear operator on $\mathfrak{H}(C)$ such that $T R=R T$.

(1) If $G-R G R^{*}$ has $\kappa$ negative squares, then there is a pair $(f, B)$, where $f \in \mathbf{S}_{0}$ and $B$ is a Blaschke product of degree $\kappa$, such that

$$
B(T) R=f(T) .
$$

(2) If there is a pair $(f, B)$ as in (1), $G-R G R^{*}$ has $\kappa^{\prime}$ negative squares for some $\kappa^{\prime} \leq \kappa$.

Remarks. (1) Let $f \in \mathbf{S}_{0}$, and define $R=f(T)$ by the $H^{\infty}$-functional calculus. Set $\tilde{f}(z)=\overline{f(\bar{z})}$. Then $R T=T R$. Since $E f(T)^{*}=E \tilde{f}\left(T^{*}\right)=\tilde{f}\left(S^{*}\right) E$, we obtain

$$
G-R G R^{*}=E^{*} E-f(T) E^{*} E f(T)^{*}=E^{*} E-E^{*} \tilde{f}\left(S^{*}\right)^{*} \tilde{f}\left(S^{*}\right) E \geq 0,
$$

because $\tilde{f}\left(S^{*}\right)$ is a contraction.

(2) It is a corollary of the theorem that if $R$ is a bounded operator on $\mathfrak{H}(C)$ such that $R T=T R$ and $G-R G R^{*} \geq 0$, then $1-R R^{*} \geq 0$. Indeed, by the theorem, $R=f(T)$ for some $f \in \mathbf{S}_{0}$, and hence $R$ is a contraction.

Proof of Theorem 3.7. We proceed as in the proof of Theorem 3.6 with the same choice of $\mathcal{V}$, data $(A, b, c)$, and set $\mathcal{D}$ of linear functionals. In exactly the same way, we show that $\mathcal{D}$ is admissible. The calculation of (2.4) is the same except for the last step. Now we have

$$
\mathcal{F}\left(x_{h}^{\prime}, x_{k}^{\prime}\right)=\langle E k, E h\rangle_{H^{2}}-\left\langle E R^{*} k, E R^{*} h\right\rangle_{H^{2}}=\left\langle\left(G-R G R^{*}\right) k, h\right\rangle_{\mathfrak{H}(C)}
$$

for all $h$ and $k$ in $\mathfrak{H}(C)$.

(1) If $G-R G R^{*}$ has $\kappa$ negative squares, so does (2.4), and hence by the Main Theorem there is a pair $(f, B)$, where $f \in \mathbf{S}_{0}$ and $B$ is a Blaschke product of degree $\kappa$, such that (2.2) holds. We use Abel summation as in the proof of Theorem [3.6 to 
show that this implies (3.4), where $B(T)$ and $f(T)$ are defined by the $H^{\infty}$-functional calculus. The commutivity of $T$ and $R$ then implies that $B(T) R=f(T)$.

(2) Reverse the steps in (1).

We turn now to problems of Loewner type, which concern boundary data. In the scalar case, solutions of interpolation problems of the type that we treat here are unique, and the results that we obtain are characterizations of boundary functions and their restrictions. Boundary values of functions in $\mathbf{S}_{\kappa}$ are understood to be computed nontangentially from inside the unit disk, and they exist a.e. on the unit circle by Fatou's Theorem.

Let $\sigma$ be normalized Lebesgue measure on $\partial \mathbf{D}:|u|=1$. When no confusion can arise, we write $L^{2}, L^{\infty}$ for $L^{2}(\sigma), L^{\infty}(\sigma)$; we identify $H^{2}$ with a subspace of $L^{2}$ in the usual way. If $\Delta$ is a measurable subset of $\partial \mathbf{D}, L^{2}(\Delta)$ is the subspace of functions in $L^{2}$ supported on $\Delta$. The characteristic function of $\Delta$ is denoted $1_{\Delta}$; if $\varphi$ is a function on $\Delta$, we view $\varphi 1_{\Delta}$ as defined on all of $\partial \mathbf{D}$ and equal to zero on the complement of $\Delta$.

We note some preliminary results from function theory.

(i) If $\varphi, \psi \in L^{2}(\Delta)$, then [29, p. 31]

$$
\begin{aligned}
\lim _{r \uparrow 1} \int_{\Delta} \int_{\Delta} \frac{\varphi(u) \overline{\psi(v)}}{1-r^{2} u \bar{v}} d \sigma(u) d \sigma(v) \\
\quad=\sum_{j=0}^{\infty}\left(\int_{\Delta} u^{j} \varphi(u) d \sigma(u)\right)\left(\int_{\Delta} v^{j} \psi(v) d \sigma(v)\right)^{-}=\left\langle Q_{-}\left(\varphi 1_{\Delta}\right), \psi 1_{\Delta}\right\rangle_{L^{2}},
\end{aligned}
$$

where $Q_{-}$is the orthogonal projection on $L^{2}$ whose range is the closed span $L_{-}^{2}$ of all functions $u^{j}, j \leq 0$. In particular, the limit on the left exists.

(ii) If $B$ is an inner function and $M_{B}$ is multiplication by $B$ on $L^{2}$, then

$$
M_{B} Q_{-} M_{B}^{*}=Q_{-}+P_{u \mathfrak{H}(B)},
$$

where $\mathfrak{H}(B)=H^{2} \ominus B H^{2}$ and $P_{u \mathfrak{H}(B)}$ is the orthogonal projection on $L^{2}$ whose range is $u \mathfrak{H}(B)$. To prove this, write $L^{2}=L_{-}^{2} \oplus u B H^{2} \oplus u \mathfrak{H}(B)$. Both sides of (3.6) coincide with the identity operator on $L_{-}^{2}$, and both coincide with the zero operator on $u B H^{2}$. It remains to check the actions of each side on elements of $L^{2}$ of the form $u h$ with $h$ in $\mathfrak{H}(B)$. This amounts to showing that $B Q_{-}(\bar{B} u h)=u h$, or equivalently, that $Q_{-}(\bar{B} u h)=\bar{B} u h$; the last equation holds by the characterization of $\mathfrak{H}(B)$ as the set of elements $h$ of $H^{2}$ such that $\bar{B} h \perp H^{2}$.

(iii) (Kronecker's Theorem) This result characterizes finite-rank Hankel operators and has several formulations, including: Let $F \in L^{\infty}$, and assume that the operator $H_{F}$ on $H^{2}$ to $L^{2} \ominus H^{2}$ which is defined as compression of multiplication by $F$ has rank $\kappa$. Then there is a Blaschke product $B$ of degree $\kappa$ such that $B F \in H^{\infty}$. For example, see Peller [27. p. 77]. Here $H_{F} h=P_{-}(F h), h \in H^{2}$, where $P_{-}$is the projection onto $L^{2} \ominus H^{2}$; equivalently, $H_{F} h=\bar{u} Q_{-}(u F h)$ where $Q_{-}$is as above.

Our first boundary result characterizes boundary functions of functions in $\mathbf{S}_{\kappa}$.

Theorem 3.8. Let $b$ and $c$ be complex-valued measurable functions on a Borel set $\Delta \subseteq \partial \mathbf{D}$ such that $c \neq 0 \sigma$-a.e. on $\Delta$. Let $\mathcal{D}$ be the set of functions $\varphi$ on $\Delta$ such that $b \varphi, c \varphi \in L^{2}(\Delta)$. If $\kappa$ is a nonnegative integer, then there is a function $S(z)$ 
in $\mathbf{S}_{\kappa}$ whose boundary function satisfies $b(u)=S(u) c(u) \sigma$-a.e. on $\Delta$ if and only if the Hermitian form

$$
L(\varphi, \psi)=\lim _{r \uparrow 1} \int_{\Delta} \int_{\Delta} \frac{c(u) \overline{c(v)}-b(u) \overline{b(v)}}{1-r^{2} u \bar{v}} \varphi(u) \overline{\psi(v)} d \sigma(u) d \sigma(v),
$$

$\varphi, \psi \in \mathcal{D}$, has $\kappa$ negative squares.

Proof. Case 1: $\partial \mathbf{D} \backslash \Delta$ is not a $\sigma$-null set.

We apply the Main Theorem with $\mathcal{V}=\{p b+q c: p, q$ polynomials $\}$. For the data $(A, b, c)$, let $A$ be multiplication by the independent variable, and let $b$ and $c$ be the given functions. We view $\mathcal{D}$ as a subspace of $\mathcal{V}^{\prime}$ by identifying each $\varphi \in \mathcal{D}$ with a linear functional on $\mathcal{V}$ by writing

$$
(f, \varphi)=\int_{\Delta} f \varphi d \sigma, \quad f \in \mathcal{V} .
$$

Condition (i) in the definition of an admissible family is easily verified, and (ii) holds because the Fourier coefficients of a function in $L^{2}$ are square summable. To check (iii), suppose $\varphi \in \mathcal{D}$ annihilates $c, A c, A^{2} c, \ldots$ Then

$$
\int_{\partial \mathbf{D}} u^{j} c \varphi 1_{\Delta} d \sigma=0, \quad j=0,1,2, \ldots
$$

Thus $c \varphi 1_{\Delta}$ belongs to $u H^{2}$. Since $\sigma(\partial \mathbf{D} \backslash \Delta)>0, c \varphi 1_{\Delta}=0 \sigma$-a.e. on $\partial \mathbf{D}$ and hence $\varphi=0 \sigma$-a.e. on $\Delta$. Therefore $\varphi$ is the zero element of $\mathcal{D}$ and hence annihilates $b, A b, A^{2} b, \ldots$ Thus $\mathcal{D}$ is admissible.

By (3.5), the Hermitian form (2.4) coincides with (3.7). An argument in [29] p. 31] shows that for any Schur functions $f$ and $B$, the identity $B(A) b=f(A) c$ is equivalent to the relation $B b=f c \sigma$-a.e. on $\Delta$.

Now suppose sq_$L=\kappa$. By part (1) of the Main Theorem, there is a pair $(f, B), f \in \mathbf{S}_{0}$ and $B$ a Blaschke product of degree $\kappa$, such that $f(A) c=B(A) b$ and hence $B b=f c \sigma$-a.e. on $\Delta$. Hence $b=S c \sigma$-a.e. on $\Delta$, where $S=f / B$ is in $\mathbf{S}_{\kappa^{\prime}}$ for some $\kappa^{\prime} \leq \kappa$. Let $S=f_{1} / B_{1}$ be a Kreŭn-Langer factorization of $S$. So $B_{1}$ is a Blaschke product of degree $\kappa^{\prime}$ (which is obtained by cancelling all of the common simple Blaschke factors in $f$ and $B$ ). Then $f_{1} c=B_{1} b \sigma$-a.e. on $\Delta$ and so $f_{1}(A) c=B_{1}(A) b$. Part (2) of the Main Theorem then gives sq_ $L \leq \kappa^{\prime}$. Therefore $\kappa=\kappa^{\prime}$ and the sufficiency part of the theorem follows.

Conversely, let $b=S c \sigma$-a.e. on $\Delta$ where $S \in \mathbf{S}_{\kappa}$. If $S=f / B$ is a Krĕ̌n-Langer factorization of $S$, then $f(A) c=B(A) b$, and by part (2) of the Main Theorem, sq_ $L=\kappa^{\prime} \leq \kappa$. By the proof of sufficiency above, $b=S_{1} c \sigma$-a.e. on $\Delta$, where $S_{1} \in \mathbf{S}_{\kappa^{\prime}}$. Since we assume that $c \neq 0 \sigma$-a.e. on $\Delta, S_{1}=b / c=S \in \mathbf{S}_{\kappa}$, and hence $L$ has $\kappa^{\prime}=\kappa$ negative squares. The necessity part of the theorem follows.

Case 2: $\Delta=\partial \mathbf{D}$.

Given $\varepsilon>0$, let $\Delta_{\varepsilon}$ be the set of points $u \in \partial \mathbf{D}$ with $\varepsilon \leq \arg u \leq 2 \pi$. Let $b_{\varepsilon}, c_{\varepsilon}$ be the restrictions of $b, c$ to $\Delta_{\varepsilon}$. Let $\mathcal{D}_{\varepsilon}$ be the set of restrictions of functions in $\mathcal{D}$ to $\Delta_{\varepsilon}$, and define $L_{\varepsilon}$ as the restriction of $L$ to $\mathcal{D}_{\varepsilon} \times \mathcal{D}_{\varepsilon}$.

If $L$ has $\kappa$ negative squares, so does $L_{\varepsilon}$ for all sufficiently small $\varepsilon$. By Case 1, for such $\varepsilon$, there is a function $S_{\varepsilon}(z)$ in $\mathbf{S}_{\kappa}$ whose boundary function satisfies $b(u)=$ $S_{\varepsilon}(u) c(u) \sigma$-a.e. on $\Delta_{\varepsilon}$. Since functions in $\mathbf{S}_{\kappa}$ are determined by their boundary values on a set of positive measure, $S_{\varepsilon}=S$ is independent of $\varepsilon$. By construction, $b(u)=S(u) c(u) \sigma$-a.e. on $\partial \mathbf{D}$. 
In the other direction, if there is an $S$ in $\mathbf{S}_{\kappa}$ whose boundary function satisfies $b(u)=S(u) c(u) \sigma$-a.e. on $\partial \mathbf{D}$, the same relation holds $\sigma$-a.e. on $\Delta_{\varepsilon}$. Again by Case $1, L_{\varepsilon}$ has $\kappa$ negative squares for all sufficiently small $\varepsilon$. Hence $L$ has $\kappa$ negative squares. Thus the result holds in the case $\Delta=\partial \mathbf{D}$.

Corollary 3.9. Let $S_{0}$ be a complex-valued measurable function on $\partial \mathbf{D}$, and let $\kappa$ be a nonnegative integer. Then there is a function $S(z)$ in $\mathbf{S}_{\kappa}$ whose boundary function satisfies $S(u)=S_{0}(u) \sigma$-a.e. on $\partial \mathbf{D}$ if and only if the Hermitian form

$$
L(\varphi, \psi)=\lim _{r \uparrow 1} \int_{\partial \mathbf{D}} \int_{\partial \mathbf{D}} \frac{1-S_{0}(u) \overline{S_{0}(v)}}{1-r^{2} u \bar{v}} \varphi(u) \overline{\psi(v)} d \sigma(u) d \sigma(v),
$$

$\varphi, S_{0} \varphi, \psi, S_{0} \psi \in L^{2}$, has $\kappa$ negative squares. When this condition is satisfied, then $\left|S_{0}(u)\right| \leq 1$-a.e. on $\partial \mathbf{D}$.

Proof. In Theorem 3.8 choose $\Delta=\partial \mathbf{D}, c=1$, and $b=S_{0}$. The last statement follows by the Kreı̆n-Langer representation (2.1) of a generalized Schur function.

We give a different version of the corollary that assumes $\left|S_{0}(u)\right| \leq 1 \sigma$-a.e. on $\partial \mathbf{D}$ as a hypothesis and then obtains additional information. Namely, the result remains true if we replace the form $L(\varphi, \psi)$ defined on $L^{2} \times L^{2}$ by (3.8) by its restriction (3.9) to $u H^{2} \times u H^{2}$.

Alternative form of Corollary [3.9. Let $S_{0}$ be a complex-valued measurable function on $\partial \mathbf{D}$ satisfying $\left|S_{0}(u)\right| \leq 1 \sigma$-a.e. on $\partial \mathbf{D}$, and let $\kappa$ be a nonnegative integer. Then there is a function $S(z)$ in $\mathbf{S}_{\kappa}$ whose boundary function satisfies $S(u)=S_{0}(u) \sigma$-a.e. on $\partial \mathbf{D}$ if and only if the Hermitian form

$$
L_{+}(\varphi, \psi)=\lim _{r \uparrow 1} \int_{\partial \mathbf{D}} \int_{\partial \mathbf{D}} \frac{1-S_{0}(u) \overline{S_{0}(v)}}{1-r^{2} u \bar{v}} \varphi(u) \overline{\psi(v)} d \sigma(u) d \sigma(v),
$$

$\varphi, \psi \in u H^{2}$, has $\kappa$ negative squares.

Proof. First assume that $S_{0} \in \mathbf{S}_{\kappa}$, or, more precisely, that $S_{0}(u)=S(u) \sigma$-a.e. for some $S(z)$ in $\mathbf{S}_{\kappa}$. We show that $\mathrm{sq}_{-} L_{+}=\kappa^{\prime}$ for some $\kappa^{\prime} \leq \kappa$. Let $S=f / B$ be a Kreĭn-Langer factorization. Then by (3.5) and (3.6), for any $\varphi, \psi \in u H^{2}$,

$$
\begin{aligned}
L_{+}(\varphi, \psi) & =\left\langle Q_{-} \varphi, \psi\right\rangle_{L^{2}}-\left\langle Q_{-}(\bar{B} f \varphi), \bar{B} f \psi\right\rangle_{L^{2}} \\
& =-\left\langle M_{B} Q_{-} M_{B}^{*} f \varphi, f \psi\right\rangle_{L^{2}} \\
& =-\left\langle\left(Q_{-}+P_{u \mathfrak{H}(B)}\right) f \varphi, f \psi\right\rangle_{L^{2}} \\
& =-\left\langle P_{u \mathfrak{H}(B)} f \varphi, f \psi\right\rangle_{L^{2}} .
\end{aligned}
$$

Since $\mathfrak{H}(B)$ has dimension $\kappa$, sq- $L_{+}=\kappa^{\prime}$ for some $\kappa^{\prime} \leq \kappa$.

Next suppose that $\mathrm{sq}_{-} L_{+}=\kappa^{\prime}$ for some $\kappa^{\prime}$. As above, for any $\varphi=u h$ and $\psi=u k$ in $u H^{2}$,

$$
L_{+}(\varphi, \psi)=\left\langle Q_{-} \varphi, \psi\right\rangle_{L^{2}}-\left\langle Q_{-} S_{0} \varphi, S_{0} \psi\right\rangle_{L^{2}}=-\left\langle Q_{-} u S_{0} h, u S_{0} k\right\rangle_{L^{2}} .
$$

Since we assume that $\mathrm{sq}_{-} L_{+}=\kappa^{\prime}$, the rank of the Hankel operator defined by $H_{S_{0}} h=\bar{u} Q_{-}\left(u S_{0} h\right), h \in H^{2}$, is $\kappa^{\prime}$. By Kronecker's theorem as stated above, there is a Blaschke product $B$ of degree $\kappa^{\prime}$ such that $B S_{0}=f$, where $f \in \mathbf{S}_{0}$ (recall that we assume that $\left|S_{0}(u)\right| \leq 1 \sigma$-a.e. on $\left.\partial \mathbf{D}\right)$. It follows that $S_{0} \in \mathbf{S}_{\kappa^{\prime \prime}}$ for some $\kappa^{\prime \prime} \leq \kappa^{\prime}$, that is, $S_{0}$ is the boundary function of a function in $\mathbf{S}_{\kappa^{\prime \prime}}$. But by the first part of the proof, we then have $\kappa^{\prime}=$ sq $_{-} L_{+} \leq \kappa^{\prime \prime}$, and so $\kappa^{\prime \prime}=\kappa^{\prime}$ and $S_{0} \in \mathbf{S}_{\kappa^{\prime}}$. 
The result follows on combining the two parts of the argument.

Example. In most cases, condition (iii) in the definition of an admissible family is trivially satisfied, but there are situations in which it does not hold. An example arises in the proof of Theorem 3.8 when $\Delta=\partial \mathbf{D}$; it is not possible to include this case in the main argument there because condition (iii) for an admissible family may fail. To see this, in the proof of Theorem 3.8 (Case 1) allow $\Delta=\partial \mathbf{D}$, and take $c=1$ and $b=\bar{u}^{\kappa}$ for some positive integer $\kappa$. An element $\varphi$ of $\mathcal{D}=L^{2}$ annihilates $c, A c, A^{2} c, \ldots$ if and only if

$$
\left(A^{j} c, \varphi\right)=\int_{\partial \mathbf{D}} u^{j} \varphi d \sigma=0, \quad j \geq 0 .
$$

This implies that

$$
\left(A^{j} b, \varphi\right)=\int_{\partial \mathbf{D}} u^{j-\kappa} \varphi d \sigma=0, \quad j \geq \kappa,
$$

but the last identity can fail for $0 \leq j<\kappa$, that is, condition (iii) in the definition of an admissible family does not hold. Nevertheless, the Hermitian form (3.7) has $\kappa$ negative squares; indeed by (3.5) for $\varphi, \psi$ in $\mathcal{D}$,

$$
\begin{aligned}
L(\varphi, \psi) & =\lim _{r \uparrow 1} \int_{\Delta} \int_{\Delta} \frac{c(u) \overline{c(v)}-b(u) \overline{b(v)}}{1-r^{2} u \bar{v}} \varphi(u) \overline{\psi(v)} d \sigma(u) d \sigma(v) \\
& =-\left\langle\left(M_{u^{\kappa}} Q_{-} M_{u^{\kappa}}^{*}-Q_{-}\right) \varphi, \psi\right\rangle_{L^{2}},
\end{aligned}
$$

where $M_{u^{\kappa}} Q_{-} M_{u^{\kappa}}^{*}-Q_{-}$is the projection of $L^{2}$ onto the span of $1, u, \ldots, u^{\kappa}$. Of course, even in this case, the conclusion of Theorem [3.8 is true as explained in Case 2 of the proof. Indeed, the identity $b(u)=S(u) c(u)$ holds $\sigma$-a.e. on $\partial \mathbf{D}$, where $S(z)=1 / z^{\kappa}$ belongs to $\mathbf{S}_{\kappa}$.

\section{INTERIOR AND BOUNDARY INTERPOLATION ON A HALF-PLANE}

Half-plane results are more simply derived by direct application of the Main Theorem than by change of variables from the disk case. A Blaschke product of degree $\kappa$ on the upper half-plane $\mathbf{C}_{+}$is a function of the form

$$
B(z)=c \prod_{j=1}^{\kappa} \frac{z-a_{j}}{z-\bar{a}_{j}},
$$

where $c$ is a constant of modulus one and $a_{1}, \ldots, a_{\kappa}$ are points in $\mathbf{C}_{+}$(not necessarily distinct). Every such function can be written as $B(z)=B_{0}((z-i) /(z+i))$, where $B_{0}(z)$ is a Blaschke product of degree $\kappa$ on the unit disk, and conversely [30]. We say that a meromorphic function $S(z)$ on $\mathbf{C}_{+}$belongs to $\mathbf{S}_{\kappa}\left(\mathbf{C}_{+}\right)$if it has the form

$$
S(z)=S_{0}\left(\frac{z-i}{z+i}\right),
$$

where $S_{0}(z)$ belongs to $\mathbf{S}_{\kappa}$ as a function on $\mathbf{D}$. If $S(z)$ belongs to $\mathbf{S}_{\kappa}\left(\mathbf{C}_{+}\right)$, then

$$
f(z)=i \frac{1+S(z)}{1-S(z)}
$$

defines a function in $\mathbf{N}_{\kappa}$, and every function in $\mathbf{N}_{\kappa}$ is obtained in this way; when $\kappa=0$ we exclude $S(z) \equiv 1$ from this correspondence. 
If $\Delta$ is a (Lebesgue) measurable subset of $(-\infty, \infty)$ and $\varphi, \psi \in L^{2}(\Delta)$, then 29 pp. 33-34]

$$
\begin{aligned}
\lim _{\epsilon \downarrow 0} \frac{i}{2} \int_{\Delta} \int_{\Delta} \frac{\varphi(s) \overline{\psi(t)}}{s-t+i \epsilon} d s d t \\
\quad=\sum_{j=0}^{\infty}\left(\int_{\Delta}\left(\frac{t-i}{t+i}\right)^{j} \frac{1}{t+i} \varphi(t) d t\right)\left(\int_{\Delta}\left(\frac{t-i}{t+i}\right)^{j} \frac{1}{t+i} \psi(t) d t\right)^{-} \\
\quad=\pi\left\langle Q_{-}\left(\varphi 1_{\Delta}\right), \psi 1_{\Delta}\right\rangle_{L^{2}(-\infty, \infty)}
\end{aligned}
$$

The meaning of $Q_{-}$here is different from that of 3.5). Let $H^{2}$ now denote the Hardy class for the upper half-plane [30. We may alternatively view $H^{2}$ as a subspace of $L^{2}(-\infty, \infty)$ by passing to boundary values. In (4.2), $Q_{-}$is the projection of $L^{2}(-\infty, \infty)$ onto the orthogonal complement of $H^{2}$ in $L^{2}(-\infty, \infty)$, or equivalently, onto the set of complex conjugates of functions in $H^{2}$, since

$$
L^{2}(-\infty, \infty)=H^{2} \oplus \overline{H^{2}} .
$$

The last equality in (4.2) follows from the fact that the functions

$$
\frac{1}{\sqrt{\pi}}\left(\frac{t-i}{t+i}\right)^{j} \frac{1}{t+i}, \quad j=0, \pm 1, \pm 2, \ldots
$$

are an orthonormal basis for $L^{2}(-\infty, \infty)$ and the part of 4.3 for $j \geq 0$ is an orthonormal basis for $H^{2}$. In particular, the infinite series in the middle term of (4.2) always converges, and the limit on the left side of 4.2) always exists.

The next result is a half-plane counterpart of Theorem 3.8 and preliminary to boundary theorems for the generalized Nevanlinna class. We understand that boundary values on $(-\infty, \infty)$ are computed nontangentially from the upper halfplane.

Theorem 4.1. Let $b$ and $c$ be complex-valued measurable functions on a Borel subset $\Delta$ of $(-\infty, \infty)$ such that $c \neq 0$ a.e. on $\Delta$. Let $\mathcal{D}$ be the set of measurable functions $\varphi$ on $\Delta$ such that $b \varphi, c \varphi \in L^{2}(\Delta)$. If $\kappa$ is a nonnegative integer, then there is a function $S(z)$ in $\mathbf{S}_{\kappa}\left(\mathbf{C}_{+}\right)$whose boundary function satisfies $b(x)=S(x) c(x)$ a.e. on $\Delta$ if and only if the Hermitian form

$$
L(\varphi, \psi)=\lim _{\varepsilon \downarrow 0} \frac{i}{2} \int_{\Delta} \int_{\Delta} \frac{c(s) \overline{c(t)}-b(s) \overline{b(t)}}{s-t+i \varepsilon} \varphi(s) \overline{\psi(t)} d s d t, \quad \varphi, \psi \in \mathcal{D},
$$

has $\kappa$ negative squares.

Proof. Case 1: $(-\infty, \infty) \backslash \Delta$ is not a Lebesgue null set.

Let $\mathcal{V}$ be the vector space of complex-valued functions on $\Delta$ of the form

$$
p\left(\frac{x-i}{x+i}\right) b(x)+q\left(\frac{x-i}{x+i}\right) c(x), \quad p, q \text { polynomials. }
$$

We identify any $\varphi \in \mathcal{D}$ with the linear functional on $\mathcal{V}$ defined by

$$
(h, \varphi)=\int_{\Delta} \frac{h(t) \varphi(t)}{t+i} d t, \quad h \in \mathcal{V} .
$$

Define data $(A, b, c)$ by choosing $A$ to be multiplication by $(x-i) /(x+i)$ and letting $b$ and $c$ be the given functions. Arguments as in the proof of Theorem 3.8 show 
that $\mathcal{D}$ is admissible; the assumption that $(-\infty, \infty) \backslash \Delta$ is not a Lebesgue null set is used to verify condition (iii) for an admissible set.

By (4.2), the Hermitian form (2.4) coincides with (4.4). We show that $f_{0}(A) c=$ $B_{0}(A) b$ for a pair $\left(f_{0}, B_{0}\right)$ of holomorphic functions which are bounded by one on the unit disk $\mathbf{D}$ if and only if $B(x) b(x)=f(x) c(x)$ a.e. on $\Delta$, where $f$ and $B$ are defined on the upper half-plane $\mathbf{C}_{+}$by

$$
\begin{aligned}
& f(z)=f_{0}\left(\frac{z-i}{z+i}\right)=\sum_{0}^{\infty} f_{j}\left(\frac{z-i}{z+i}\right)^{j}, \\
& B(z)=B_{0}\left(\frac{z-i}{z+i}\right)=\sum_{0}^{\infty} B_{j}\left(\frac{z-i}{z+i}\right)^{j} .
\end{aligned}
$$

If $f_{0}(A) c=B_{0}(A) b$, then for every $\varphi \in \mathcal{D}$,

$$
\sum_{j=0}^{\infty} f_{j}\left(A^{j} c, \varphi\right)=\sum_{j=0}^{\infty} B_{j}\left(A^{j} b, \varphi\right)
$$

that is,

$$
\sum_{j=0}^{\infty} f_{j} \int_{\Delta} \frac{1}{t+i}\left(\frac{t-i}{t+i}\right)^{j} c(t) \varphi(t) d t=\sum_{j=0}^{\infty} B_{j} \int_{\Delta} \frac{1}{t+i}\left(\frac{t-i}{t+i}\right)^{j} b(t) \varphi(t) d t .
$$

By the arbitrariness of $\varphi$, on summing the series in the Abel sense as in [29. p. 32], we obtain

$$
f_{0}\left(\frac{x-i}{x+i}\right) c(x)=B_{0}\left(\frac{x-i}{x+i}\right) b(x)
$$

and hence $f(x) c(x)=B(x) b(x)$ a.e. on $\Delta$. These steps are reversible, and the assertion follows.

To complete the proof in Case 1, we apply the Main Theorem as in the proof of Theorem 3.8,

Case 2: $\Delta=(-\infty, \infty)$.

We reduce this to Case 1 in the same way as in the proof of Theorem 3.8.

An indefinite extension of the Generalized Loewner Theorem [29, p. 34] follows as an immediate consequence of Theorem 4.1 .

Theorem 4.2. Let $f_{0}$ be a complex-valued measurable function defined on a Borel subset $\Delta$ of $(-\infty, \infty)$ such that $f_{0}+i \neq 0$ a.e. on $\Delta$. Let $\mathcal{D}$ be the set of measurable functions $\varphi$ on $\Delta$ such that $\varphi, f_{0} \varphi \in L^{2}(\Delta)$. If $\kappa$ is a nonnegative integer, there exists a function $f \in \mathbf{N}_{\kappa}$ such that $f=f_{0}$ a.e. on $\Delta$ if and only if the Hermitian form

$$
L(\varphi, \psi)=\lim _{\varepsilon \downarrow 0} \int_{\Delta} \int_{\Delta} \frac{f_{0}(s)-\overline{f_{0}(t)}}{s-t+i \varepsilon} \varphi(s) \overline{\psi(t)} d s d t, \quad \varphi, \psi \in \mathcal{D},
$$

has $\kappa$ negative squares.

The assumption that $f_{0}+i \neq 0$ a.e. on $\Delta$ does not restrict the generality: it is automatically satisfied if $f=f_{0}$ a.e. on $\Delta$ for some $f \in \mathbf{N}_{\kappa}$. For if $f(z)$ and $S(z)$ are related by (4.1), then $|S(x)| \leq 1$ by the Kreinn-Langer representation (2.1), and hence $\operatorname{Im} f_{0}(x) \geq 0$ a.e. on $\Delta$. 
Proof. We apply Theorem 4.1 with $b=f_{0}-i$ and $c=f_{0}+i$. With this choice of $b$ and $c$, the set of Hermitian forms $L$ in Theorems 4.1 and 4.2 coincide. By Theorem 4.1, sq_ $L=\kappa$ if and only if there is a function $S(z)$ in $\mathbf{S}_{\kappa}\left(\mathbf{C}_{+}\right)$such that $b(x)=S(x) c(x)$ a.e. on $\Delta$, that is, $f_{0}(x)-i=S(x)\left[f_{0}(x)+i\right]$ or

$$
f_{0}(x)=i \frac{1+S(x)}{1-S(x)}
$$

a.e. on $\Delta$. The result thus follows from the correspondence (4.1) between $\mathbf{S}_{\kappa}\left(\mathbf{C}_{+}\right)$ and $\mathbf{N}_{\kappa}$.

The preceding half-plane results can be recast in a different form using Hilbert transforms. The connection comes from equation (4.2) and the formula [30, p. 113]

$$
Q_{-}=(I+i H) / 2
$$

for the projection onto the orthogonal complement of $H^{2}$ in $L^{2}(-\infty, \infty)$. Here $H$ denotes the Hilbert transform, which is defined for any $\varphi$ in $L^{2}(-\infty, \infty)$ by the principal-value integral

$$
(H \varphi)(x)=P V \frac{1}{\pi} \int_{-\infty}^{\infty} \frac{\varphi(t)}{t-x} d t=\lim _{\epsilon \downarrow 0} \frac{1}{\pi} \int_{|t-x|>\epsilon} \frac{\varphi(t)}{t-x} d t .
$$

The limit exists pointwise a.e. on the real line and also in the metric of $L^{2}(-\infty, \infty)$. The compression of $H$ to $L^{2}(\Delta)$ for any measurable subset $\Delta$ of $(-\infty, \infty)$ is denoted $H_{\Delta}$ : if $\varphi \in L^{2}(\Delta)$, then

$$
\left(H_{\Delta} \varphi\right)(x)=P V \frac{1}{\pi} \int_{\Delta} \frac{\varphi(t)}{t-x} d t=\lim _{\epsilon \downarrow 0} \frac{1}{\pi} \int_{|t-x|>\epsilon} \frac{\varphi(t)}{t-x} d t \quad \text { a.e. on } \Delta,
$$

where the limit is in the metric of $L^{2}(\Delta)$. The operators $i H$ and $i H_{\Delta}$ are selfadjoint (in particular, $H_{\Delta}^{*}=-H_{\Delta}$ ), and $i H$ is also unitary. By the convolution theorem ([29, p. 35], [30, p. 111]), for any $\varphi, \psi \in L^{2}(\Delta) \cap L^{\infty}(\Delta)$,

$$
H_{\Delta}\left[\varphi\left(H_{\Delta} \psi\right)+\left(H_{\Delta} \varphi\right) \psi\right]=\left(H_{\Delta} \varphi\right)\left(H_{\Delta} \psi\right)-\varphi \psi \quad \text { a.e. on } \Delta \text {. }
$$

Lemma 4.3. Let $f_{0}$ be a complex-valued measurable function defined on a Borel subset $\Delta$ of $(-\infty, \infty)$ such that $f_{0}+i \neq 0$ a.e. on $\Delta$. Let $\mathcal{D}$ be the set of measurable functions $\varphi$ on $\Delta$ such that $\varphi, f_{0} \varphi \in L^{2}(\Delta)$. If $\kappa$ is a nonnegative integer, there exists a function $f \in \mathbf{N}_{\kappa}$ such that $f=f_{0}$ a.e. on $\Delta$ if and only if the Hermitian form

$$
L(\varphi, \psi)=\pi\left\langle\left(H_{\Delta}-i I\right)\left(f_{0} \varphi\right), \psi\right\rangle_{L^{2}(\Delta)}+\pi\left\langle\varphi,\left(H_{\Delta}-i I\right)\left(f_{0} \psi\right)\right\rangle_{L^{2}(\Delta)},
$$

$\varphi, \psi \in \mathcal{D}$, has $\kappa$ negative squares. The conclusion remains true if $\mathcal{D}$ is replaced by the set $\mathcal{E}=\mathcal{D} \cap L^{\infty}(\Delta)$.

Proof. Combining (4.2) and (4.6), we obtain

$$
\lim _{\varepsilon \downarrow 0} \int_{\Delta} \int_{\Delta} \frac{\varphi(s) \overline{\psi(t)}}{s-t+i \varepsilon} d s d t=\pi\left\langle\left(H_{\Delta}-i I\right) \varphi, \psi\right\rangle_{L^{2}(\Delta)}, \quad \varphi, \psi \in L^{2}(\Delta) .
$$

It follows that the form $L$ in Theorem 4.2 is given by (4.7), and so the first statement of the lemma follows from Theorem 4.2.

To prove the last statement, it is enough to show that for any $\varphi$ and $\psi$ in $\mathcal{D}$, there are sequences $\varphi_{n}$ and $\psi_{n}$ in $\mathcal{E}$ such that $L\left(\varphi_{n}, \psi_{n}\right) \rightarrow L(\varphi, \psi)$, since this implies that the number of negative squares of $L$ does not decrease when it is restricted to $\mathcal{E} \times \mathcal{E}$. In fact, let $\varphi_{n}(t)=\varphi(t)$ or 0 according as $|\varphi(t)| \leq n$ or $|\varphi(t)|>n$, and define 
$\psi_{n}(t)$ similarly $(n=1,2, \ldots)$. Then $\varphi_{n}, \psi_{n} \in \mathcal{E}$ for all $n$, and $\varphi_{n} \rightarrow \varphi, \psi_{n} \rightarrow \psi$ in the metric of $L^{2}(\Delta)$. Hence also $f_{0} \varphi_{n} \rightarrow f_{0} \varphi, f_{0} \psi_{n} \rightarrow f_{0} \psi$ in the metric of $L^{2}(\Delta)$, and so $L\left(\varphi_{n}, \psi_{n}\right) \rightarrow L(\varphi, \psi)$ because $H_{\Delta}$ is a continuous operator on $L^{2}(\Delta)$.

We can convert the limit in (4.5) to a different form when $f_{0}$ is real valued.

Theorem 4.4. Let $f_{0}$ be a real-valued measurable function defined on a Borel subset $\Delta$ of $(-\infty, \infty)$. Let $\mathcal{D}$ be the set of measurable functions $\varphi$ on $\Delta$ such that $\varphi, f_{0} \varphi \in L^{2}(\Delta)$. If $\kappa$ is a nonnegative integer, there exists a function $f \in \mathbf{N}_{\kappa}$ such that $f=f_{0}$ a.e. on $\Delta$ if and only if the Hermitian form

$$
L(\varphi, \psi)=\lim _{\varepsilon \downarrow 0} \iint_{|t-s|>\epsilon} \frac{f_{0}(s)-f_{0}(t)}{s-t} \varphi(s) \overline{\psi(t)} d s d t, \quad \varphi, \psi \in \mathcal{D},
$$

has $\kappa$ negative squares.

Integration in (4.9) is over the set $(\Delta \times \Delta) \cap\{(s, t):|t-s|>\epsilon\}$.

Proof. As in [29, p. 37], for any $\varphi, \psi$ in $\mathcal{D}$ we have

$$
\begin{aligned}
\lim _{\varepsilon \downarrow 0} \iint_{|t-s|>\epsilon} \frac{f_{0}(s)-f_{0}(t)}{s-t} \varphi(s) \overline{\psi(t)} d s d t \\
=\lim _{\varepsilon \downarrow 0} \int_{\Delta}\left(\int_{|t-s|>\epsilon} \frac{f_{0}(s) \varphi(s)}{s-t} d s\right) \overline{\psi(t)} d t \\
\quad \quad-\lim _{\varepsilon \downarrow 0} \int_{\Delta}\left(\int_{|t-s|>\epsilon} \frac{\varphi(s)}{s-t} d s\right) f_{0}(t) \overline{\psi(t)} d t \\
=\pi\left\langle H_{\Delta}\left(f_{0} \varphi\right), \psi\right\rangle_{L^{2}(\Delta)}-\pi\left\langle H_{\Delta} \varphi, f_{0} \psi\right\rangle_{L^{2}(\Delta)} \\
=\pi\left\langle H_{\Delta}\left(f_{0} \varphi\right), \psi\right\rangle_{L^{2}(\Delta)}+\pi\left\langle\varphi, H_{\Delta}\left(f_{0} \psi\right)\right\rangle_{L^{2}(\Delta)} .
\end{aligned}
$$

Since $f_{0}$ is real-valued, we can rewrite this in the form (4.7), and therefore the assertion follows from Lemma 4.3 .

In the definite case, Theorem 4.4 has a dual result for functions having pure imaginary values [29, pp. 39-41]. A full generalization of the dual result to the class $\mathbf{N}_{\kappa}$ is unknown, but we are able to present some partial information. For simplicity, we assume that the given function $g_{0}$ and the set $\Delta$ are bounded.

Theorem 4.5. Let $g_{0}$ be a bounded real-valued measurable function on a bounded Borel subset $\Delta$ of $(-\infty, \infty)$. Let $\kappa$ be a nonnegative integer.

(1) There is a function $f \in \mathbf{N}_{\kappa}$ such that $f=-H_{\Delta} g_{0}$ a.e. on $\Delta$ if and only if the Hermitian form

$$
L(\varphi, \psi)=\pi \int_{\Delta}\left[\varphi \bar{\psi}-\left(H_{\Delta} \varphi\right)\left(\overline{H_{\Delta} \psi}\right)\right] g_{0} d t, \quad \varphi, \psi \in L^{\infty}(\Delta),
$$

has $\kappa$ negative squares.

(2) If the condition in (1) holds and $g_{0} \geq 0$ a.e. on $\Delta$, then there is a function $h \in \mathbf{N}_{\kappa^{\prime}}, \kappa^{\prime} \leq \kappa$, such that $h=i g_{0}$ a.e. on $\Delta$.

Proof. (1) We apply Lemma 4.3 with the real-valued function $f_{0}=-H_{\Delta} g_{0}$. Since $g_{0}$ and $\Delta$ are bounded, $f_{0} \in L^{2}(\Delta)$ and the set $\mathcal{E}$ in Lemma 4.3 is $\mathcal{E}=L^{\infty}(\Delta)$. As 
in [29, p. 39], for any $\varphi$ and $\psi$ in $L^{\infty}(\Delta)$, (4.7) is given by

$$
\begin{aligned}
L(\varphi, \psi) & =\pi\left\langle\left(H_{\Delta}-i I\right)\left(f_{0} \varphi\right), \psi\right\rangle_{L^{2}(\Delta)}+\pi\left\langle\varphi,\left(H_{\Delta}-i I\right)\left(f_{0} \psi\right)\right\rangle_{L^{2}(\Delta)} \\
& =\pi\left\langle H_{\Delta}\left(f_{0} \varphi\right), \psi\right\rangle_{L^{2}(\Delta)}+\pi\left\langle\varphi, H_{\Delta}\left(f_{0} \psi\right)\right\rangle_{L^{2}(\Delta)} \\
& =-\pi\left\langle f_{0} \varphi, H_{\Delta} \psi\right\rangle_{L^{2}(\Delta)}-\pi\left\langle H_{\Delta} \varphi, f_{0} \psi\right\rangle_{L^{2}(\Delta)} \\
& =-\pi \int_{\Delta}\left[f_{0} \varphi\left(H_{\Delta} \bar{\psi}\right)+\left(H_{\Delta} \varphi\right) f_{0} \bar{\psi}\right] d t \\
& =\pi \int_{\Delta}\left[\varphi\left(H_{\Delta} \bar{\psi}\right)+\left(H_{\Delta} \varphi\right) \bar{\psi}\right] H_{\Delta} g_{0} d t \\
& =-\pi \int_{\Delta}\left\{H_{\Delta}\left[\varphi\left(H_{\Delta} \bar{\psi}\right)+\left(H_{\Delta} \varphi\right) \bar{\psi}\right]\right\} g_{0} d t \\
& =\pi \int_{\Delta}\left[\varphi \bar{\psi}-\left(H_{\Delta} \varphi\right)\left(\overline{\left.H_{\Delta} \psi\right)}\right] g_{0} d t .\right.
\end{aligned}
$$

We have shown that (4.7) coincides with (4.10) on $\mathcal{E} \times \mathcal{E}$, and therefore the result follows from Lemma 4.3.

(2) Assume the condition in (1) holds and $g_{0} \geq 0$ a.e. on $\Delta$. Let $f_{0}=-H_{\Delta} g_{0}$ a.e. on $\Delta$. Then by the first part of the theorem, there is a function $f \in \mathbf{N}_{\kappa}$ such that $f(x+i 0)=f_{0}(x)$ a.e. on $\Delta$. Define $h(z)$ and $k(z)$ in the upper half-plane by

$$
h(z)=k(z)+f(z)=\frac{1}{\pi} \int_{\Delta} \frac{g_{0}(t)}{t-z} d t+f(z) .
$$

Since $g_{0} \geq 0$ a.e. on $\Delta, k(z)$ has nonnegative imaginary part on the upper half-plane. Hence the kernel

$$
\frac{h(z)-\overline{h(\zeta)}}{z-\bar{\zeta}}=\frac{k(z)-\overline{k(\zeta)}}{z-\bar{\zeta}}+\frac{f(z)-\overline{f(\zeta)}}{z-\bar{\zeta}}
$$

has $\kappa^{\prime} \leq \kappa$ negative squares, that is, $h(z)$ belongs to $\mathbf{N}_{\kappa^{\prime}}$. The boundary function $k_{0}(x)=k(x+i 0)$ of $k(z)$ can be computed from [29, Theorem A, p. 35]:

$$
k_{0}(x)=i\left[g_{0}(x)-i\left(H_{\Delta} g_{0}\right)(x)\right] \quad \text { a.e. on } \Delta .
$$

Therefore $h_{0}(x)=h(x+i 0)$ is given by

$$
h_{0}(x)=k_{0}(x)+f_{0}(x)=i\left[g_{0}(x)-i\left(H_{\Delta} g_{0}\right)(x)\right]-\left(H_{\Delta} g_{0}\right)(x)=i g_{0}(x)
$$

a.e. on $\Delta$.

\section{Proof of the main Result}

We use the Ball and Helton almost-commutant lifting theorem [11. This result has been extended and refined in [9], 18]. Our statement follows [9, Theorem 1.1], which is slightly more precise than the original. In our applications, it is sufficient to take the underlying Kreĭn spaces to be Hilbert spaces. A Hilbert space isometry $W \in \mathfrak{L}(\mathfrak{G})$ is called an isometric dilation of an operator $T \in \mathfrak{L}(\mathfrak{H})$ if $\mathfrak{G}=\mathfrak{H} \oplus \mathfrak{K}$ for some Hilbert space $\mathfrak{K}$, and

$$
W=\left[\begin{array}{ll}
T & 0 \\
* & *
\end{array}\right]
$$

relative to the decomposition $\mathfrak{G}=\mathfrak{H} \oplus \mathfrak{K}$. If $H$ is a selfadjoint operator on a Hilbert space, ind $H$ is the dimension of the spectral subspace of $H$ corresponding to the interval $(-\infty, 0)$. 
Theorem 5.1. For each $j=1,2$, let $T_{j} \in \mathfrak{L}\left(\mathfrak{H}_{j}\right)$ be a contraction on the Hilbert space $\mathfrak{H}_{j}$, let $W_{j} \in \mathfrak{L}\left(\mathfrak{G}_{j}\right)$ be an isometric dilation of $T_{j}$ on a Hilbert space $\mathfrak{G}_{j}$, and let $P_{j}$ be the projection of $\mathfrak{G}_{j}$ onto $\mathfrak{H}_{j}$. Let $C \in \mathfrak{L}\left(\mathfrak{H}_{1}, \mathfrak{H}_{2}\right)$ be an operator such that $C T_{1}=T_{2} C$.

(1) If ind $\left(1-C^{*} C\right)=\kappa$, then there exists a pair $(\mathcal{E}, \widetilde{C})$ such that $\mathcal{E}$ is a closed $W_{1}$-invariant subspace of $\mathfrak{G}_{1}$ of codimension equal to $\kappa$ and $\widetilde{C}$ is a contraction operator on $\mathcal{E}$ into $\mathfrak{G}_{2}$ satisfying

$$
\left.\widetilde{C} W_{1}\right|_{\mathcal{E}}=W_{2} \widetilde{C} \quad \text { and } \quad P_{2} \widetilde{C}=\left.C P_{1}\right|_{\mathcal{E}} .
$$

(2) If there is a pair $(\mathcal{E}, \widetilde{C})$ with the properties in $(1)$, then ind $\left(1-C^{*} C\right) \leq \kappa$.

In part (1), the subspace $\mathcal{E}$ can be chosen to be any closed $W_{1}$-invariant subspace of $\mathfrak{G}_{1}$ of codimension equal to $\kappa$ such that $\left.C P_{1}\right|_{\mathcal{E}}$ is a contraction [9].

Proof of the Main Theorem. Let $S$ be multiplication by $z$ on $H^{2}$. By parts (i) and (ii) of Definition 2.1 the set of functions $h_{x^{\prime}}(z)=\sum_{j=0}^{\infty}\left(A^{j} c, x^{\prime}\right) z^{j}, x^{\prime} \in \mathcal{D}$, is a linear subspace $\mathfrak{H}_{c}$ of $H^{2}$ which is invariant under $S^{*}$. By part (iii) of Definition 2.1 the formula

$$
X_{0}: \sum_{j=0}^{\infty}\left(A^{j} c, x^{\prime}\right) z^{j} \rightarrow \sum_{j=0}^{\infty}\left(A^{j} b, x^{\prime}\right) z^{j}
$$

defines a linear operator $X_{0}$ from $\mathfrak{H}_{c}$ into $H^{2}$.

(1) Assume $\mathcal{F}$ has $\kappa$ negative squares. We show that this assumption implies that $X_{0}=Y_{0}+K$, where $Y_{0}$ is a contraction and $K$ is a finite-rank operator, and hence $X_{0}$ is bounded in the norm of $H^{2}$. To do this, let $\mathfrak{X}$ be $\mathfrak{H}_{c}$ as a vector space equipped with the linear and symmetric inner product

$$
\left\langle h_{x^{\prime}}, h_{y^{\prime}}\right\rangle_{\mathfrak{X}}=\mathcal{F}\left(x^{\prime}, y^{\prime}\right), \quad x^{\prime}, y^{\prime} \in \mathcal{D} .
$$

Since $\mathcal{F}$ has $\kappa$ negative squares, $\mathfrak{X}$ contains a $\kappa$-dimensional subspace $\mathfrak{N}$ which is the antispace of a Hilbert space, and no $(\kappa+1)$-dimensional subspace of $\mathfrak{X}$ has this property [6, Lemma 1.1.1']. Writing

$$
\mathfrak{N}^{\perp}=\left\{h \in \mathfrak{X}:\langle h, k\rangle_{\mathfrak{X}}=0 \text { for all } k \in \mathfrak{N}\right\},
$$

we claim that

$$
\mathfrak{X}=\mathfrak{N}+\mathfrak{N}^{\perp} \quad \text { and } \quad \mathfrak{N} \cap \mathfrak{N}^{\perp}=\{0\} .
$$

If $h \in \mathfrak{N} \cap \mathfrak{N}^{\perp}$, then $\langle h, h\rangle_{\mathfrak{X}}=0$; this implies $h=0$ because $h \in \mathfrak{N}$ and the $\mathfrak{X}$-inner product is definite on $\mathfrak{N}$. To see that $\mathfrak{X}=\mathfrak{N}+\mathfrak{N}^{\perp}$, choose a complete orthogonal set $h_{1}, \ldots, h_{\kappa}$ in $\mathfrak{N}$ (in the $\mathfrak{X}$-inner product). Then any $h \in \mathfrak{X}$ can be written as

$$
h=\sum_{j=1}^{\kappa} \frac{\left\langle h, h_{j}\right\rangle_{\mathfrak{X}}}{\left\langle h_{j}, h_{j}\right\rangle_{\mathfrak{X}}} h_{j}+\left(h-\sum_{j=1}^{\kappa} \frac{\left\langle h, h_{j}\right\rangle_{\mathfrak{X}}}{\left\langle h_{j}, h_{j}\right\rangle_{\mathfrak{X}}} h_{j}\right) .
$$

The first term here belongs to $\mathfrak{N}$ and the second is in $\mathfrak{N}^{\perp}$; so $\mathfrak{X}=\mathfrak{N}+\mathfrak{N}^{\perp}$ and the claim is proved. Thus $\mathfrak{N}^{\perp}$ is a subspace of $\mathfrak{X}$ having a finite-dimensional complement in $\mathfrak{X}$. For any $h \in \mathfrak{N}^{\perp}$, by the definitions of $\mathcal{F}$ and the $\mathfrak{X}$-inner product,

$$
0 \leq\langle h, h\rangle_{\mathfrak{X}}=\langle h, h\rangle_{H^{2}}-\left\langle X_{0} h, X_{0} h\right\rangle_{H^{2}} .
$$

Hence $\left.X_{0}\right|_{\mathfrak{N} \perp}$ is a contraction in the norm of $H^{2}$. We are now able to construct operators $Y_{0}$ and $K$ with the required properties. First consider $\mathfrak{N}^{\perp}$ as a linear manifold of $H^{2}$ and let $X_{0}^{\prime}: \mathfrak{N}^{\perp} \rightarrow H^{2}$ be the restriction of $X_{0}$ to $\mathfrak{N}^{\perp}$. Let $\widehat{X}_{0}$ 
be the everywhere defined contraction on $H^{2}$ which coincides with $X_{0}^{\prime}$ on $\mathfrak{N}^{\perp}$ and is 0 on the orthogonal complement of $\mathfrak{N}^{\perp}$ in $H^{2}$. Then $Y_{0}$ is defined to be the restriction of $\widehat{X}_{0}$ to $\mathfrak{H}_{c}$. By this construction, $Y_{0}$ is a contraction on $\mathfrak{H}_{c}$ into $H^{2}$, and $Y_{0}$ agrees with $X_{0}$ on $\mathfrak{N}^{\perp}$. Now set $K=X_{0}-Y_{0}$. By (5.1) the range of $K$ is the range of $\left.K\right|_{\mathfrak{N}}$, which has finite dimension. We have shown that $X_{0}=Y_{0}+K$, where $Y_{0}$ is a contraction and $K$ has finite rank. In particular, $X_{0}$ is bounded in the norm of $H^{2}$. Let $X$ be the extension by continuity of $X_{0}$ to the closure of $\mathfrak{H}_{c}$ in $H^{2}$.

We apply Theorem 5.1 with these choices:

$$
\begin{aligned}
& \mathfrak{H}_{1}=H^{2} \text { and } T_{1}=S ; \\
& \mathfrak{H}_{2}=\overline{\mathfrak{H}}_{c}\left(\text { closure in } H^{2}\right) \text { and } T_{2}=E_{2}^{*} S E_{2}, \text { where } E_{2}: \mathfrak{H}_{2} \rightarrow H^{2} \text { is inclusion; } \\
& \mathfrak{G}_{1}=\mathfrak{G}_{2}=H^{2} \text { and } W_{1}=W_{2}=S ; \\
& \left.C=X^{*} \text { (as an operator from } H^{2} \text { to } \mathfrak{H}_{2}\right) .
\end{aligned}
$$

Trivially, $W_{1}$ is an isometric dilation of $T_{1}$. We show that $W_{2}$ is an isometric dilation of $T_{2}$. Since $S^{*} \mathfrak{H}_{c} \subseteq \mathfrak{H}_{c}, S^{*} \mathfrak{H}_{2} \subseteq \mathfrak{H}_{2}$. Hence the matrix decomposition of $S^{*}$ relative to the decomposition $H^{2}=\mathfrak{H}_{2} \oplus \mathfrak{H}_{2}^{\perp}$ has the form

$$
S^{*}=\left[\begin{array}{cc}
E_{2}^{*} S^{*} E_{2} & * \\
0 & *
\end{array}\right],
$$

which is equivalent to

$$
W_{2}=S=\left[\begin{array}{cc}
E_{2}^{*} S E_{2} & 0 \\
* & *
\end{array}\right]=\left[\begin{array}{cc}
T_{2} & 0 \\
* & *
\end{array}\right] .
$$

It is not hard to see that $C T_{1}=T_{2} C$ and ind $-\left(1-C C^{*}\right)=$ ind $_{-}\left(1-X^{*} X\right)=\kappa$; since always ind $-\left(1-C^{*} C\right)=$ ind $_{-}\left(1-C C^{*}\right)$, we have ind $\left(1-C^{*} C\right)=\kappa$. By Theorem 5.1 (1), there is a pair $(\mathcal{E}, \widetilde{C})$ such that

(i) $\mathcal{E}$ is a closed subspace of $H^{2}$ of codimension $\kappa$ which is invariant under $S$,

(ii) $\widetilde{C}: \mathcal{E} \rightarrow H^{2}$ is a contraction,

(iii) $P_{\mathfrak{H}_{2}} \widetilde{C}=\left.X^{*}\right|_{\mathcal{E}}$,

(iv) $\left.\widetilde{C} S\right|_{\mathcal{E}}=S \widetilde{C}$.

For any function $F(z)$ in $\mathbf{S}_{0}$, write $\widetilde{F}(z)=\overline{F(\bar{z})}$. By $(\mathrm{i}), \mathcal{E}=\widetilde{B} H^{2}$, where $B(z)$ is a Blaschke product of degree $\kappa$. For any $\varphi \in \mathbf{S}_{0}$, let $M_{\varphi}$ be the operator multiplication by $\varphi$ on $H^{2}$. We show that $\widetilde{C} M_{\widetilde{B}}$ commutes with $S$. In fact, for each $h \in H^{2}$, $\widetilde{B} h \in \mathcal{E}$ and

$$
\widetilde{C} M_{\widetilde{B}} S h=\widetilde{C} S(\widetilde{B} h) \stackrel{(i v)}{=} S \widetilde{C} \widetilde{B} h=S \widetilde{C} M_{\widetilde{B}} h .
$$

Since $\widetilde{C} M_{\widetilde{B}}$ is a contraction by (ii), $\widetilde{C} M_{\widetilde{B}}=M_{\tilde{f}}$ for some $f \in \mathbf{S}_{0}$. For any $x^{\prime} \in \mathcal{D}$ and $h \in H^{2}$,

$$
\begin{array}{r}
\left\langle\sum_{j=0}^{\infty}\left(A^{j} c, x^{\prime}\right) z^{j}, M_{\tilde{f}} h\right\rangle_{H^{2}}=\left\langle\sum_{j=0}^{\infty}\left(A^{j} c, x^{\prime}\right) z^{j}, \widetilde{C} M_{\widetilde{B}} h\right\rangle_{H^{2}} \\
=\left\langle\sum_{j=0}^{\infty}\left(A^{j} c, x^{\prime}\right) z^{j}, P_{\mathfrak{H}_{2}} \widetilde{C} M_{\widetilde{B}} h\right\rangle_{H^{2}} \stackrel{(i i i)}{=}\left\langle\sum_{j=0}^{\infty}\left(A^{j} c, x^{\prime}\right) z^{j}, X^{*} M_{\widetilde{B}} h\right\rangle_{H^{2}} \\
=\left\langle\sum_{j=0}^{\infty}\left(A^{j} b, x^{\prime}\right) z^{j}, \widetilde{B} h\right\rangle_{H^{2}} .
\end{array}
$$


Choosing $h=1$, we obtain

$$
\left\langle\sum_{j=0}^{\infty}\left(A^{j} c, x^{\prime}\right) z^{j}, \tilde{f}\right\rangle_{H^{2}}=\left\langle\sum_{j=0}^{\infty}\left(A^{j} b, x^{\prime}\right) z^{j}, \widetilde{B}\right\rangle_{H^{2}}, \quad x^{\prime} \in \mathcal{D} .
$$

Thus (2.3) and hence (2.2) hold. This completes the proof of part (1) of the Main Theorem.

(2) Assume that (2.2) holds with $f$ in $\mathbf{S}_{0}$ and $B$ a Blaschke product of degree $\kappa$. We show that this assumption implies that the operator $X_{0}$ defined at the beginning of the proof is bounded. Define $\tilde{f}$ and $\widetilde{B}$ as in part (1) above. The assumption (2.2) can be rewritten in the form (5.3). Hence for all $x^{\prime} \in \mathcal{D}$,

$$
\begin{gathered}
\left\langle z \tilde{f}, \sum_{j=0}^{\infty}\left(A^{j} c, x^{\prime}\right) z^{j}\right\rangle_{H^{2}}=\left\langle\tilde{f}, S^{*}\left(\sum_{j=0}^{\infty}\left(A^{j} c, x^{\prime}\right) z^{j}\right)\right\rangle_{H^{2}} \\
=\left\langle\tilde{f}, \sum_{j=0}^{\infty}\left(A^{j} c, A^{\prime} x^{\prime}\right) z^{j}\right\rangle_{H^{2}} \stackrel{5.3}{=}\left\langle\widetilde{B}, \sum_{j=0}^{\infty}\left(A^{j} b, A^{\prime} x^{\prime}\right) z^{j}\right\rangle_{H^{2}} \\
=\left\langle z \widetilde{B}, \sum_{j=0}^{\infty}\left(A^{j} b, x^{\prime}\right) z^{j}\right\rangle_{H^{2}} .
\end{gathered}
$$

Writing as before $h_{x^{\prime}}(z)=\sum_{j=0}^{\infty}\left(A^{j} c, x^{\prime}\right) z^{j}$ for all $x^{\prime} \in \mathcal{D}$, we can extend this relation to

$$
\left\langle\tilde{f} h, h_{x^{\prime}}\right\rangle_{H^{2}}=\left\langle\widetilde{B} h, X_{0} h_{x^{\prime}}\right\rangle_{H^{2}},
$$

first for every polynomial $h$ and then for every $h$ in $H^{2}$. Now the boundedness of $X_{0}$ follows from the fact that $\mathcal{E}=\widetilde{B} H^{2}$ has codimension $\kappa$ in $H^{2}$. Let $X$ be the extension by continuity of $X_{0}$ to the closure $\mathfrak{H}_{2}$ of $\mathfrak{H}_{c}$ in $H^{2}$. Since

$$
\mathcal{F}\left(x^{\prime}, y^{\prime}\right)=\left\langle h_{x^{\prime}}, h_{y^{\prime}}\right\rangle_{H^{2}}-\left\langle X h_{x^{\prime}}, X h_{y^{\prime}}\right\rangle_{H^{2}}, \quad x^{\prime}, y^{\prime} \in \mathcal{D},
$$

to show that (2.4) has $\kappa^{\prime}$ negative squares for some $\kappa^{\prime} \leq \kappa$, it is sufficient to show that ind $-\left(1-X^{*} X\right)$ is at most $\kappa$. Write

$$
X=\left[\begin{array}{l}
X_{1} \\
X_{2}
\end{array}\right]
$$

relative to the decomposition $H^{2}=\mathcal{E} \oplus \mathcal{E}^{\perp}$. By (5.4), $X^{*} M_{\widetilde{B}}=P_{\mathfrak{H}_{2}} M_{\tilde{f}}$ is a contraction. Therefore $X_{1}^{*}=\left.X^{*}\right|_{\mathcal{E}}$ and $X_{1}$ are contractions. Thus

$$
1-X^{*} X=\left(1-X_{1}^{*} X_{1}\right)-X_{2}^{*} X_{2},
$$

where $1-X_{1}^{*} X_{1} \geq 0$ and $-X_{2}^{*} X_{2}$ has rank at most $\kappa$. Hence ind $-\left(1-X^{*} X\right) \leq \kappa$ and part (2) of the Main Theorem follows.

We restate the Main Theorem in a way which characterizes all solutions of Nudel'man's problem in terms of the solutions of the corresponding lifting problem. In the statement of this result, we identify two pairs $\left(f_{1}, B_{1}\right)$ and $\left(f_{2}, B_{2}\right)$ such that $f_{1}=\gamma f_{2}$ and $B_{1}=\gamma B_{2}$ for some constant $\gamma,|\gamma|=1$, and we adopt notation as in the proof of the Main Theorem. In particular, $S$ is multiplication by $z$ on $H^{2}$. For any $\varphi \in \mathbf{S}_{0}$, let $M_{\varphi}$ be multiplication by $\varphi$ on $H^{2}$. We write $\tilde{\varphi}(z)=\sum_{0}^{\infty} \bar{\varphi}_{n} z^{n}$ if $\varphi(z)=\sum_{0}^{\infty} \varphi_{n} z^{n}$. 
Main Theorem (Alternative Form). Let $(A, b, c)$ be given data, $\mathcal{D}$ an admissible set. Define $\mathcal{F}$ on $\mathcal{D} \times \mathcal{D}$ by (2.4), and let $\kappa$ be a nonnegative integer. Then

$$
\mathrm{sq}_{-} \mathcal{F} \leq \kappa
$$

if and only if there is a pair $(f, B)$, where $f \in \mathbf{S}_{0}$ and $B$ is a Blaschke product of degree $\kappa$, such that $f(A) c=B(A) b$. The set of all such pairs $(f, B)$ is in one-to-one correspondence with the set of all pairs $(\mathcal{E}, \widetilde{C})$ such that

(i) $\mathcal{E}$ is a closed subspace of $H^{2}$ of codimension $\kappa$ which is invariant under $S$;

(ii) $\widetilde{C}: \mathcal{E} \rightarrow H^{2}$ is a contraction operator;

(iii) if $P_{\mathcal{E}}$ is the projection onto $\mathcal{E}$, then for all $x^{\prime} \in \mathcal{D}$,

$$
\widetilde{C}^{*}: \sum_{j=0}^{\infty}\left(A^{j} c, x^{\prime}\right) z^{j} \rightarrow P_{\mathcal{E}}\left\{\sum_{j=0}^{\infty}\left(A^{j} b, x^{\prime}\right) z^{j}\right\}
$$

(iv) $\left.\widetilde{C} S\right|_{\mathcal{E}}=S \widetilde{C}$.

The correspondence is determined by the relations $\mathcal{E}=\widetilde{B} H^{2}$ and $\widetilde{C} M_{\widetilde{B}}=M_{\tilde{f}}$.

Proof. If sq_ $\mathcal{F}=\kappa^{\prime} \leq \kappa$, then by part (1) of the Main Theorem there is a pair $\left(f_{1}, B_{1}\right)$, where $f_{1} \in \mathbf{S}_{0}$ and $B_{1}$ is a Blaschke product of degree $\kappa^{\prime}$, such that $f_{1}(A) c=B_{1}(A) b$. If $\kappa^{\prime}=\kappa$, the pair $(f, B)=\left(f_{1}, B_{1}\right)$ has the required properties. Otherwise write $\kappa=\kappa^{\prime}+r$ and let $(f(z), B(z))=\left(z^{r} f_{1}(z), z^{r} B_{1}(z)\right)$. Then $f \in \mathbf{S}_{0}$ and $B$ is a Blaschke product of degree $\kappa$. If

$$
f_{1}(z)=\sum_{j=0}^{\infty} \alpha_{j} z^{j} \quad \text { and } \quad B_{1}(z)=\sum_{j=0}^{\infty} \beta_{j} z^{j}
$$

then the coefficients of $f(z)$ consist of $r$ zeros followed by $\alpha_{0}, \alpha_{1}, \ldots$, and similarly for $B(z)$. In order to prove that $f(A) c=B(A) b$, we must therefore show that for all $x^{\prime} \in \mathcal{D}$,

$$
\sum_{j=0}^{\infty} \alpha_{j}\left(A^{j+r} c, x^{\prime}\right)=\sum_{j=0}^{\infty} \beta_{j}\left(A^{j+r} b, x^{\prime}\right) .
$$

By the invariance of $\mathcal{D}$ under $A^{\prime}$, this identity is equivalent to

$$
\sum_{j=0}^{\infty} \alpha_{j}\left(A^{j} c,\left(A^{\prime}\right)^{r} x^{\prime}\right)=\sum_{j=0}^{\infty} \beta_{j}\left(A^{j} b,\left(A^{\prime}\right)^{r} x^{\prime}\right)
$$

which holds because $f_{1}(A) c=B_{1}(A) b$. This proves the necessity part of the first statement. Sufficiency follows from part (2) of the Main Theorem.

Given a pair $(f, B)$ where $f \in \mathbf{S}_{0}$ and $B$ is a Blaschke product of degree $\kappa$ such that $f(A) c=B(A) b$, define $(\mathcal{E}, \widetilde{C})$ by $\mathcal{E}=\widetilde{B} H^{2}$ and $\widetilde{C} M_{\widetilde{B}}=M_{\tilde{f}}$. Obviously (i) and (ii) hold, and we easily check (iv). To prove (iii), we use the identity $f(A) c=B(A) b$ to show that

$$
\left\langle\sum_{j=0}^{\infty}\left(A^{j} c, x^{\prime}\right) z^{j}, \tilde{f} h\right\rangle_{H^{2}}=\left\langle\sum_{j=0}^{\infty}\left(A^{j} b, x^{\prime}\right) z^{j}, \widetilde{B} h\right\rangle_{H^{2}}
$$

first for monomials $h(z)=z^{k}$ and hence for all $h \in H^{2}$. Therefore, for all $x^{\prime} \in \mathcal{D}$ and $h \in H^{2}$,

$$
\left\langle\sum_{j=0}^{\infty}\left(A^{j} c, x^{\prime}\right) z^{j}, \widetilde{C} \widetilde{B} h\right\rangle_{H^{2}}=\left\langle\sum_{j=0}^{\infty}\left(A^{j} b, x^{\prime}\right) z^{j}, \widetilde{B} h\right\rangle_{H^{2}},
$$


and since $\mathcal{E}=\widetilde{B} H^{2}$, this implies (iii).

Conversely, if $(\mathcal{E}, \widetilde{C})$ is a pair that satisfies (i)-(iv), the proof of the Main Theorem shows that $\mathcal{E}=\widetilde{B} H^{2}$ and $\widetilde{C} M_{\widetilde{B}}=M_{\tilde{f}}$, where $f \in \mathbf{S}_{0}$ and $B$ is a Blaschke product of degree $\kappa$, such that $f(A) c=B(A) b$. The correspondence is one-to-one because the relation $\mathcal{E}=\widetilde{B} H^{2}$ determines $B$ up to a constant factor of modulus one.

\section{REFERENCES}

[1] V. M. Adamjan, D. Z. Arov, and M. G. Krĕn, Analytic properties of the Schmidt pairs of a Hankel operator and the generalized Schur-Takagi problem, Mat. Sb. (N.S.) 86 (128) (1971), 34-75. MR 45:7505

[2] D. Alpay, V. Bolotnikov, and A. Dijksma, On the Nevanlinna-Pick interpolation problem for generalized Stieltjes functions, Integral Equations Operator Theory 30 (1998), no. 4, 379-408. MR 99k:47032

[3] D. Alpay, V. Bolotnikov, A. Dijksma, and J. Rovnyak, Some extensions of Loewner's theory of monotone operator functions, J. Funct. Anal., to appear.

[4] D. Alpay, T. Constantinescu, A. Dijksma, and J. Rovnyak, Notes on interpolation in the generalized Schur class. I. Applications of realization theory, Oper. Theory Adv. Appl., Birkhäuser, Basel, to appear.

[5] D. Alpay, A. Dijksma, and H. Langer, On the Loewner problem in the class $N_{\kappa}$, Proc. Amer. Math. Soc. 130 (2002), 2057-2066.

[6] D. Alpay, A. Dijksma, J. Rovnyak, and H. S. V. de Snoo, Schur functions, operator colligations, and reproducing kernel Pontryagin spaces, Oper. Theory Adv. Appl., vol. 96, Birkhäuser, Basel, 1997. MR 2000a:47024

[7] D. Alpay and J. Rovnyak, Loewner's theorem for kernels having a finite number of negative squares, Proc. Amer. Math. Soc. 127 (1999), no. 4, 1109-1117. MR 99m:47013

[8] A. Amirshadyan and V. Derkach, Interpolation in generalized Nevanlinna and Stieltjes classes, J. Operator Theory 42 (1999), no. 1, 145-188. MR 2000e:47026

[9] R. Arocena, T. Ya. Azizov, A. Dijksma, and S. A. M. Marcantognini, On commutant lifting with finite defect. II, J. Funct. Anal. 144 (1997), no. 1, 105-116. MR 98a:47006

[10] J. A. Ball, I. Gohberg, and L. Rodman, Interpolation of rational matrix functions, Oper. Theory Adv. Appl., vol. 45, Birkhäuser, Basel, 1990. MR 92m:47027

[11] J. A. Ball and J. W. Helton, A Beurling-Lax theorem for the Lie group $U(m, n)$ which contains most classical interpolation theory, J. Operator Theory 9 (1983), no. 1, 107-142. MR 84m:47046

[12] V. Bolotnikov and H. Dym, On degenerate interpolation, entropy and extremal problems for matrix Schur functions, Integral Equations Operator Theory 32 (1998), no. 4, 367-435. MR 99m:47014

[13] T. Constantinescu and A. Gheondea, On the indefinite trigonometric moment problem of I. S. Iohvidov and M. G. Krě̀n, Math. Nachr. 171 (1995), 79-94. MR 95m:47019

[14] - On the Carathéodory type problem of M. G. Kreı̆n and H. Langer, C. R. Acad. Sci. Paris Sér. I Math. 327 (1998), no. 3, 243-247. MR 99i:47025

[15] L. de Branges and J. Rovnyak, Square summable power series, Holt, Rinehart and Winston, New York, 1966. MR 35:5909

[16] A. Dijksma and H. Langer, Notes on a Nevanlinna-Pick interpolation problem for generalized Nevanlinna functions, Topics in interpolation theory (Leipzig, 1994), Oper. Theory Adv. Appl., vol. 95, Birkhäuser, Basel, 1997, pp. 69-91. MR 98g:47015

[17] C. Foias, A. E. Frazho, and M. A. Kaashoek, A weighted version of almost commutant lifting, Systems, approximation, singular integral operators and related topics, Oper. Theory Adv. Appl., vol. 129, Birkhäuser, Basel, 2001, pp. 311-340.

[18] A. Gheondea, Contractive intertwining dilations of quasi-contractions, Z. Anal. Anwendungen 15 (1996), no. 1, 31-44. MR 97e:47030

[19] L. B. Golinskiı̌, A generalization of the matrix Nevanlinna-Pick problem, Izv. Akad. Nauk Armyan. SSR Ser. Mat. 18 (1983), no. 3, 187-205, English transl., Soviet J. Contemporary Math. Anal. 18 (1983), no. 3, 22-39; private English translation by V. Katsnelson. MR 85g:47049 
[20] S. Hassi, H. de Snoo, and H. Woracek, Some interpolation problems of Nevanlinna-Pick type. The Krĕn-Langer method, Contributions to operator theory in spaces with an indefinite metric (Vienna, 1995), Oper. Theory Adv. Appl., vol. 106, Birkhäuser, Basel, 1998, pp. 201216. MR 2001c: 47022

[21] J. W. Helton, Orbit structure of the Möbius transformation semigroup acting on $H^{\infty}$ (broadband matching), Topics in functional analysis (essays dedicated to M. G. Kreĭn on the occasion of his 70th birthday), Academic Press, New York, 1978, pp. 129-157. MR 81e:46019

[22] T. S. Ivanchenko, The Nevanlinna-Pick problem in the case of an indefinite metric, Dokl. Akad. Nauk Ukrain. SSR Ser. A (1980), no. 5, 8-13, 92. MR 84i:47056

[23] M. G. Kreĭn and H. Langer, Über die verallgemeinerten Resolventen und die charakteristische Funktion eines isometrischen Operators im Raume $\Pi_{\kappa}$, Hilbert space operators and operator algebras (Proc. Internat. Conf., Tihany, 1970), North-Holland, Amsterdam, 1972, pp. 353399. Colloq. Math. Soc. János Bolyai, 5. MR 54:11103

[24] _ Über einige Fortsetzungsprobleme, die eng mit der Theorie hermitescher Operatoren im Raume $\Pi_{\kappa}$ zusammenhängen. I. Einige Funktionenklassen und ihre Darstellungen, Math. Nachr. 77 (1977), 187-236. MR 57:1173

[25] A. A. Nudel'man, A new problem of the type of the moment problem, Dokl. Akad. Nauk SSSR 233 (1977), no. 5, 792-795, English transl., Soviet Math. Dokl. 18 (1977), 507-510. MR 57:10379

[26] (1981), no. 4, 790-793. MR 82f:30033

[27] V. V. Peller, An excursion into the theory of Hankel operators, Holomorphic Spaces (S. Axler, J. E. McCarthy, and D. Sarason, eds.), MSRI Publications, vol. 33, Cambridge University Press, Cambridge, 1998, pp. 65-120. MR 99e:47033

[28] G. Popescu, Meromorphic interpolation in several variables, preprint, 2001.

[29] M. Rosenblum and J. Rovnyak, Hardy classes and operator theory, Oxford Mathematical Monographs, Oxford University Press, New York, 1985, Dover republication, New York, 1997. MR 97j:47002

[30] _ Topics in Hardy classes and univalent functions, Birkhäuser Advanced Texts: Basler Lehrbücher, Birkhäuser, Basel, 1994. MR 97a:30047

[31] A. L. Sakhnovich, Modification of V. P. Potapov's scheme in the indefinite case, Matrix and operator valued functions, Oper. Theory Adv. Appl., vol. 72, Birkhäuser, Basel, 1994, pp. 185-201. MR 96b:47016

[32] D. Sarason, Generalized interpolation in $H^{\infty}$, Trans. Amer. Math. Soc. 127 (1967), 179-203. MR 34:8193

[33] _ Sub-Hardy Hilbert spaces in the unit disk, John Wiley \& Sons Inc., New York, 1994. MR 96k:46039

[34] _ Nevanlinna-Pick interpolation with boundary data, Integral Equations Operator Theory 30 (1998), no. 2, 231-250. MR 98m:47017

[35] M. Schreiber, A functional calculus for general operators in Hilbert space, Trans. Amer. Math. Soc. 87 (1958), 108-118. MR 20:6040

[36] B. Sz.-Nagy and C. Foias, Harmonic analysis of operators on Hilbert space, North-Holland Publishing Co., Amsterdam, 1970. MR 43:947

Department of Mathematics, Ben-Gurion University of the Negev, P.O. Box 653, 84105 BeER-SHEVA, ISRAEL

E-mail address: dany@math.bgu.ac.il

Programs in Mathematical Sciences, University of Texas at Dallas, Box 830688, Richardson, TEXAS 75083-0688

E-mail address: tiberiu@utdallas.edu

Department of Mathematics, University of Groningen, P.O. Box 800, 9700 AV GroninGen, The Netherlands

E-mail address: dijksma@math.rug.nl

University of Virginia, Department of Mathematics, P.O. Box 400137, CharlottesVILle, Virginia 22904-4137

E-mail address: rovnyak@Virginia.EDU 